\title{
An Evolutionary Framework for Automatic and Guided Discovery of Algorithms
}

\author{
Ruchira Sasanka \\ Intel Corporation \\ ruchira.sasanka@intel.com
}

\begin{abstract}
This paper presents Automatic Algorithm Discoverer (AAD), an evolutionary framework for synthesizing programs of high complexity. To guide evolution, prior evolutionary algorithms have depended on fitness (objective) functions, which are challenging to design. To make evolutionary progress, instead, AAD employs Problem Guided Evolution (PGE), which requires introduction of a group of problems together. With PGE, solutions discovered for simpler problems are used to solve more complex problems in the same group. PGE also enables several new evolutionary strategies, and naturally yields to High-Performance Computing (HPC) techniques.

We find that PGE and related evolutionary strategies enable AAD to discover algorithms of similar or higher complexity relative to the state-of-the-art. Specifically, AAD produces Python code for 29 array/vector problems ranging from min, max, reverse, to more challenging problems like sorting and matrix-vector multiplication. Additionally, we find that $\mathrm{AAD}$ shows adaptability to constrained environments/inputs and demonstrates outside-of-the-box problem solving abilities.
\end{abstract}

Keywords Synthesis, Evolution, Python, HPC

\section{Introduction}

Program synthesis involves automatically assembling a program from simpler components. It is analogous to searching the entire space created by all possible permutations of those components, looking for solutions that satisfy given requirements. Many such search strategies (such as enumerative, deduction-based, constraint-solving, stochastic) have been proposed to address this challenge [3, 12, 32, 34, 41, 47, 49].

In this work, we propose an evolution-based search strategy, implemented in the Automatic Algorithm Discoverer $(A A D)$. AAD can synthesize programs of relatively high complexity (including loops, nested blocks, nested function calls, etc.), based on a subset of Python as grammar, and can generate executable Python code. In this paper we use AAD to discover algorithmic solutions to array/vector problems.

Evolutionary algorithms use a fitness (objective) function to pick the fittest individuals from a population $[23,25,27$, 28]. The traits of the fittest individuals can recombine (crossover) to form the next generation. However, designing an effective fitness function could be challenging for complex problems $[9,17,20]$. We propose an alternative way to guide

\author{
Konstantinos Krommydas \\ Intel Corporation \\ konstantinos.krommydas@intel.com
}

evolution without a fitness function, by adding several potentially related problems together into a group. We call this Problem Guided Evolution (PGE) and it is analogous to the way we teach children to solve complex problems. For instance, to help discover an algorithm for finding the area of a polygon, we may ask a student to find a way to calculate the area of a triangle. That is, simpler problems guide solutions to more complex ones. Notably, PGE does not require knowing the exact algorithm nor the exact constituents to a solution, but rather a few potentially related problems. In AAD, PGE allows more complex solutions to be derived through (i) composition (combining simpler ones), and through (ii) mutation of alredy discovered ones.

Grouping related problems for PGE, like designing a fitness function, is not automatic and currently requires human insight. However, PGE could be a more natural way to attack complex problems. As a concrete example, Figure 1 shows code that AAD produced in order to sort a non-empty array in ascending order (SortAsc). To solve this, we grouped ten potentially related problems together to guide evolution: min, max, first/last index, reverse, remove first/last, is-in-array, and sort ascending/descending. AAD used solutions it found itself for some of those problems to discover an algorithm for sorting: by first finding the minimum of the input array, appending that minimum to a new list, removing the minimum from the input array, and repeating these steps until the entire input array is processed. Though not the most elegant nor the most efficient implementation, a machine being able to discover an algorithm for sorting starting from a basic subset of Python illustrates the capabilities of AAD and the utility of PGE.

Overall, this paper makes the following contributions:

- Use of Problem Guided Evolution to eliminate objective functions in evolutionary algorithms.

- Use of multiple evolutionary strategies (such as diverse environments \& solutions, cross-pollination, and ganged evolution), and evaluation of their effectiveness via a wide range of experiments.

- Application of AAD to solve 29 array/vector problems in general-purpose Python language, demonstrating evolutionary algorithms are capable of solving complex state-of-the-art problems.

- Support of loops to discover algorithms that can accept any (non-zero) input size. 


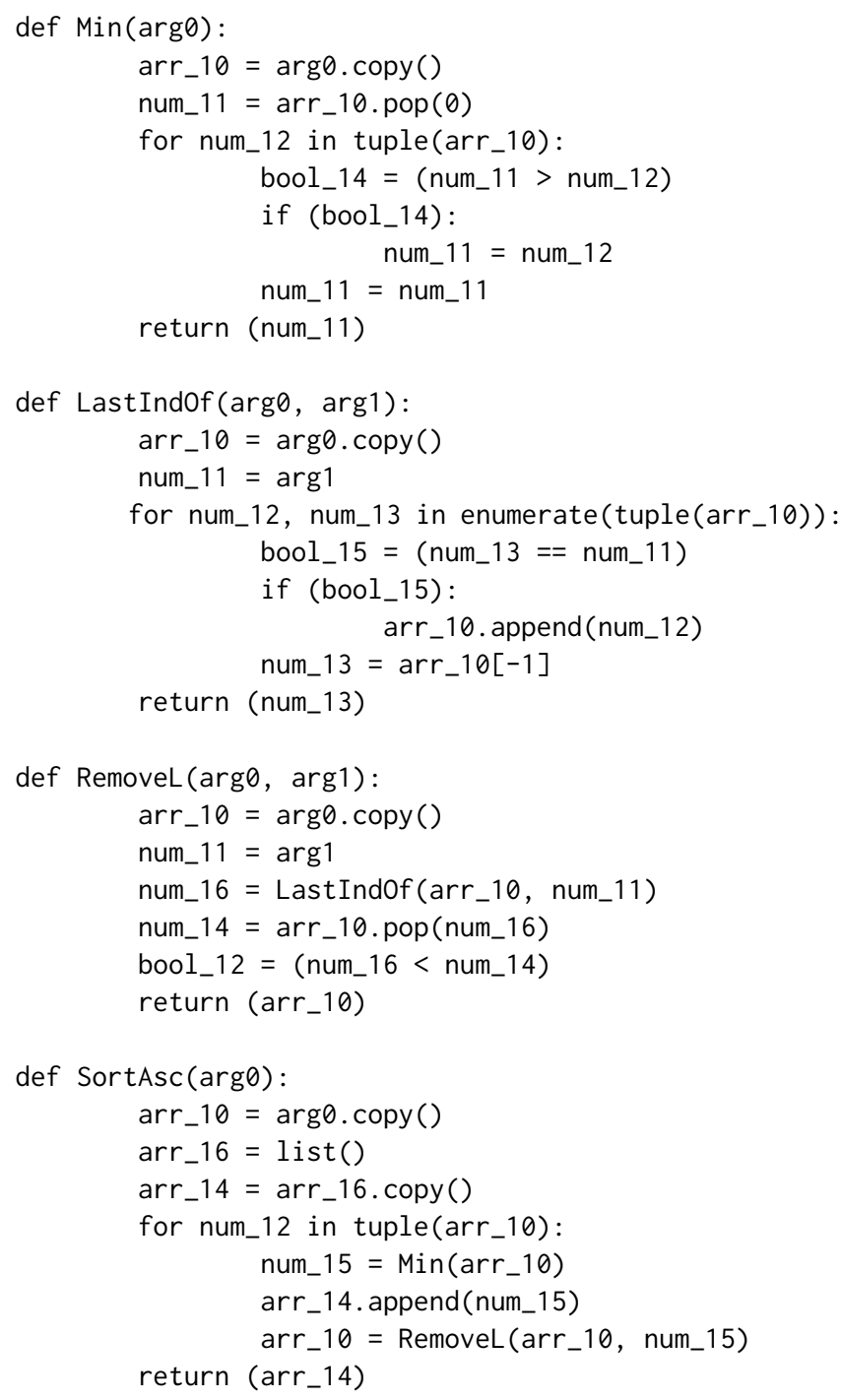

Figure 1. AAD synthesized algorithm for sorting

- Mapping of inherently parallel evolutionary process to HPC hardware and techniques.

We find that PGE and related evolutionary strategies are effective at discovering solutions to our array/vector problems. Among other findings, notable is the adaptability of $\mathrm{AAD}$ to constrained environments and inputs, as well as its ability to find creative solutions.

The rest of the paper is organized as follows: In Section 2 we present the design details of AAD. Specifically, we discuss: (i) the three constituent parts of AAD, with special emphasis on the Evolver and its three phases that construct the solution, (ii) the evolutionary strategies employed by AAD and similarities to biological evolution, and (iii) engineering challenges we faced and their solutions, as well as our HPCoriented approach in the AAD implementation. Section 3 presents our experimental setup and Section 4 discusses the

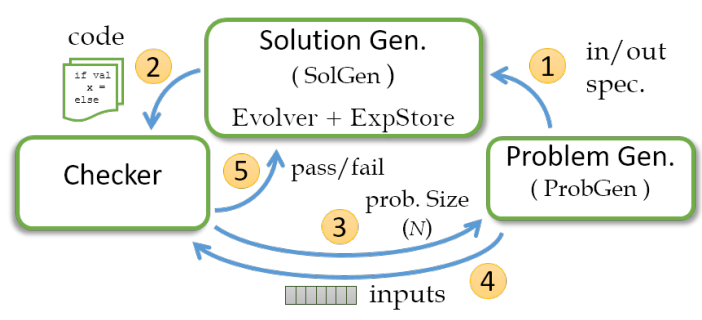

Figure 2. Components of AAD.

results of putting $\mathrm{AAD}$ in test in a broad range of experiments encompassing a variety of problems. Last, we present related work (Section 5), a discussion and future work (Section 6), and conclude the paper in Section 7.

\section{Design}

As shown in Figure 2, AAD consists of three components: (i) a Problem Generator (ProbGen) to generate a problem, (ii) a Solution Generator (SolGen) to come up with potential solutions (programs), and (iii) a Checker to check those solutions.

\subsection{Problem Generator (ProbGen)}

Each problem we want solved starts with its own ProbGen. ProbGen is responsible for: (i) specifying the number and types of inputs and outputs, and (ii) generating inputs for a given problem size. For instance, for maximum finding (Max), ProbGen specifies that Max takes one array as its input and produces a number as its output. In addition, when requested to generate inputs for a problem of size $N$, it produces an input array filled with $N$ numbers.

\subsection{Checker}

Checker is responsible for accepting or rejecting a solution generated for a given problem. Checker executes the generated program with input(s) generated by ProbGen, and produces output. The Checker contains logic to either accept or reject the output, as in [21]. Therefore, a Checker is specific to a given ProbGen, and both go hand in hand.

A Checker does not necessarily need an implementation of the algorithm it seeks to discover, although some problems do require an alternative implementation. For instance, the Checker for problem "Sort" does not have to sort the input array. Rather, it can compare each two adjacent elements in the output array and see whether the two elements are in the expected order. As soon as it detects an out of order pair, it can declare failure. If each pair of elements is in order, and the output array contains exactly the same elements as the input array, which can be checked by removing matching elements, the Checker can accept the solution.

For some problems based on the physical world, the input and output data may be found out empirically. For instance, to develop an algorithm that can predict future temperatures at a specific place, historical temperature data may be used 
or data may be gathered using sensors. In other words, the physical world can function as a Checker for some of the models (algorithms) we want to discover.

\subsection{The Solution Generator (SolGen)}

SolGen primarily consists of two components: (i) an Expression/Idiom Store, and (ii) an Evolver.

\subsubsection{Expression/Idiom Store (ExpStore)}

SolGen constructs source programs using a grammar, as in $[2,15,31,50]$. The subset of Python grammar AAD uses is stored in ExpStore, and is given in Table 1.

In $\mathrm{AAD}$, grammar rules are augmented with type information, as in [13, 36, 39]. AAD supports four types: numbers (NUM), Boolean (BOOL), arrays (ARR), and arrays-of-arrays (AoA), which can model matrices. Further, each operand of an expression is marked as a Consumer (read-only), a Producer (write-only), or a ProdCon (read-modify-write). With this additional information, a typical binary addition rule becomes:

$$
\text { NUM (Prod) }=\text { NUM (Cons) + NUM (Cons) }
$$

In Table 1, the producer operands are italicized and ProdCon operands are underlined. The rest is all consumers. Some auxiliary grammar rules (e.g., for concatenating statements, function declarations) are omitted for brevity.

When AAD uses a statement with a BLOCK (a code block), it inserts at least one randomly selected expression into the BLOCK as a heuristic. We call such a construct an idiom. In addition, when an If Stmt is inserted, an additional expression producing a BOOL is inserted before it, to make sure that there is an expression producing a BOOL for the If Stmt to consume. Consequently, every For Stmt and If Stmt is inserted as an idiom. Reduction is another such idiom. Idioms allow for faster construction of useful programs.

In ExpStore, the operands of expressions are generally not given any identifier names. However, in idioms, if two expressions have a producer-consumer relationship (e.g., BOOL expression in If idiom), we assign the common operand a common integer identifier to link the producer and the consumer.

For Stmts allow us to iterate over two types of data structures - ARR or AoA. The latter type is used in a contextsensitive way - if and only if the function has a parameter of type AoA. For each of those two types, AAD allows enumerated and non-enumerated for-loops. In Python, enumerated iterative loops provide both the index and the item at that index, making them more versatile. However, such a statement leads to two producers, one for the element and one for the index.

In $\mathrm{AAD}$, the Expr class representing expressions are structured so that it can represent multiple Producers, Consumers and/or ProdCons. A function is modeled as a sequence of Expr objects.

\begin{tabular}{|c|c|}
\hline Expr/Stmt & Representation \\
\hline Arithmetic & $\begin{array}{l}N U M=\text { NUM op NUM } \\
\text { op }=+,-, *, / /\end{array}$ \\
\hline Compare & $\begin{array}{l}\text { BOOL }=\text { BOOL op BOOL } \\
\text { op }=<,>,==,<=,>=, !=\end{array}$ \\
\hline Head/Tail & $N U M=\mathrm{ARR}[\mathbf{0}] \mid \mathrm{ARR}[\mathbf{- 1}]$ \\
\hline Pop (Head/Tail) & $\begin{array}{l}N U M=\text { ARR.pop }(0) \\
N U M=\text { ARR.pop }()\end{array}$ \\
\hline Pop at Ind & $N U M=$ ARR.pop(NUM) \\
\hline Append & ARR.append() \\
\hline New Array & $\overline{A R R}=$ list () \\
\hline Constant & $N U M=0 \mid 1$ \\
\hline Array copy & $A R R=$ ARR.copy () \\
\hline AoA copy & $A o A=\operatorname{AoA} \cdot \operatorname{copy}()$ \\
\hline Func Arg & $A N Y \_T Y P E=$ param \\
\hline Assign Stmt & $N U M=\mathrm{NUM}$ \\
\hline Return Stmt & return $A N Y \_T Y P E$ \\
\hline Reduction & $\underline{\mathrm{NUM}}+=\mathrm{NUM}$ \\
\hline If Stmt & 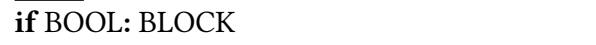 \\
\hline For Stmt & $\begin{array}{l}\text { for } N U M \text { in } A R R: B L O C K \\
\text { for } N U M, N U M \text { in enumerate(ARR): BLOCK } \\
\text { for } A R R \text { in } A O A: B L O C K \\
\text { for } N U M, A R R \text { in enumerate(AoA): } B L O C K\end{array}$ \\
\hline BLOCK & BEGIN Statements END \\
\hline
\end{tabular}

Table 1. Expressions and statements used in AAD. ANY_TYPE represents any of NUM, ARR, BOOL, or AoA. Token param represents a function parameter.

For a BLOCK, we add BEGIN and END delimiters (dummy Expr objects), which are useful in analysis. They are not emitted as part of generated Python code, though they decide indentation of emitted code.

Since function calls are expressions, ExpStore can include calls to library functions. However, to be as minimalistic as possible, we use library calls needed only for basic array access - pop head/tail or a given index, and append to tail.

At first glance, ProdCon operands may appear as an unnecessary complication. However, Python's library functions like append() modify the source operand, and so does the $R e$ duction expression in Table 1. ProdCon operands allow modeling these succinctly. In program synthesis such operands provide a less obvious but important benefit because they reduce the total number of operands in an expression and reduce the number of unique producers in a program, leading to a reduction of search space.

More expressions and statements can be added to ExpStore as needed, but only those we use for current results are listed in Table 1. Especially, the grammar shown leads to regular control-flow and avoids statements like break and continue, though addition of these is readily supported.

\subsubsection{Evolver}

The Evolver is responsible for combining expressions and idioms to assemble a program (a function), which can potentially solve the problem presented by ProbGen. The Evolver constructs the solution function (SolFunc) in three phases. 


\section{Phase 1: Building of SolFunc}

First, Evolver initializes SolFunc so that the input argument expressions are at the top of the function and the return statement is at the bottom of the function as shown in Figure 3. The building of SolFunc boils down to inserting items in ExpStore between the input arguments and the return statement. Second, the Evolver builds SolFunc bottom-up, starting from the return statement. If the return statement consumes a value of type $T$, the Evolver randomly picks an expression, $E$, from ExpStore that has a producer (or a ProdCon) of type $T$ and inserts it above the return statement. Now, $E$, has its own consumer operands, for which producers must be found. Consequently, the Evolver picks another expression at random from ExpStore to produce each source operand of $E$, and inserts it randomly somewhere above $E$, but below function arguments. Instead of selecting an expression, the Evolver may randomly choose to insert an idiom from the ExpStore.

If input arguments are only of one type, $T$, just below the input arguments, the Evolver inserts an expression consuming an operand of type $T$ and producing a type other than $T$. For instance, if the sole input argument is an array, the Evolver inserts a randomly picked expression that consumes an array and produces a number. This heuristic ensures that values of both types, ARR and NUM, are generated at the top of the function, allowing any later expressions to consume values of these two common types. Additionally, local copies are made of incoming ARR function parameters to prevent them from being modified within the function (see Figure 3).

\section{Phase 2: Linking Producers and Consumers}

In Phase 1, Evolver gives each producer operand a unique integer ID, when an expression is inserted into SolFunc. In Phase 2, consumer (and ProdCon) operands have to be assigned one of those IDs, linking a producer and a consumer. The Evolver, starts this process at the bottom of SolFunc, starting with the return statement. The return statement, $R$, has only one consumer of type $T$. The Evolver looks for all expressions above $R$, for one producing an operand of type $T$. Out of all those expressions, the Evolver picks one at random and assigns the ID of that producer (or ProdCon) to the consumer, thereby linking the two. The Evolver continues this process, from bottom to top of SolFunc, until every consumer operand is linked with a producer (or ProdCon). One producer can be consumed by one or more consumers (or ProdCons).

Since AAD supports block nesting, while linking producers and consumers Evolver has to make sure scoping rules are met. For instance, in Figure 3, producer num_13 is in an inner block than its consumer, the return statement, violating scoping rules. There are several ways to fix this and we chose to alias num_13 with another producer (e.g., num_11) at the same level as the consumer, causing to emit num_11

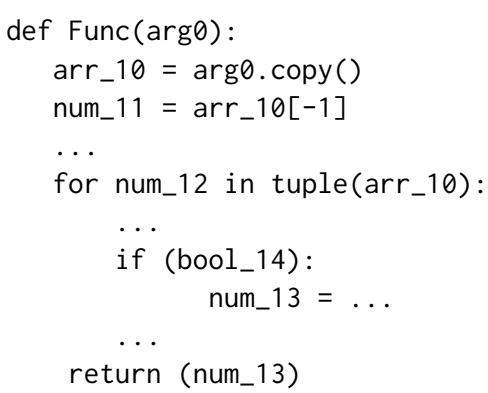

Figure 3. Linking producers and consumers

instead of num_13, for all operands in all expressions. If there are multiple consumers of a producer, the producer must be at the same level as the outermost consumer.

The linking phase also opportunistically detects dead expressions, which are those with producers that are not consumed. A rigorous attempt is not made to detect and remove all dead code because Python interpreter executing the produced code can do this for itself.

At the end of Phase 2, SolFunc is complete and can be compiled and executed.

\section{Phase 3: Operator \& Function Call Mutation}

The completed SolFunc can be optionally mutated in Phase 3. The first four expressions in Table 1 are designed to capture multiple operations. For instance, the first grammar rule for binary Arithmetic operations captures four operations: +, -, *, // (integer division).

As a result, we can easily change an expression from an addition to a multiplication without re-building SolFunc or re-linking producers and consumers. In Phase 3, the Evolver randomly changes these operations. In addition to operators, Phase 3 can mutate an existing function call (e.g., Max) to another compatible call (e.g., Min), with the same type of argument(s) and the return type.

\subsection{Checking Output}

Once SolFunc is built (and mutated), it is executed to produce output using Python's exec() function. The output is checked with the Checker, which either accepts or rejects the output. If the first output is accepted, the same SolFunc is tested with more inputs of different sizes, generated using ProbGen. If all those tests are accepted by the Checker, the SolFunc is declared a solution for the problem. The above three phases constitute one evolutionary step.

\subsection{Evolutionary Strategies}

This section describes evolutionary strategies used by AAD.

\subsubsection{Composition}

AAD uses self-discovered solutions to simpler problems to compose more complex solutions. To this end, AAD evolves 
an entire group of problems at once, as shown in Figure 4(a). Once an acceptable SolFunc is found for one problem in the group, it is allowed to be called by others by adding an appropriate function call to ExpStore. Since a function call is an expression, when a SolFunc is accepted by the Checker, $\mathrm{AAD}$ creates a function call expression for it with appropriate parameters. AAD uses the input-output description given by ProbGen to determine the type of each parameter and the return type. By AAD's convention, the input parameters are always read-only (consumers). However, a function like Remove (ARR, NUM) modifies the first parameter. We allow such functions to be created as well by allowing ProbGen to identify the first parameter as a ProdCon and omit a separate return value (i.e., omit a separate producer). When emitting, $\mathrm{AAD}$ emits such a function with the same identifier for the return value and the first argument as arr1 = Remove (arr1, num1).

Function composition has a profound effect on reducing the size of search space. For instance, assume we allow $N$ statements in SolFunc and the ExpStore contains $E$ items to pick from. Since each statement in SolFunc can be filled in $E$ ways (with repetition), there are $E^{N}$ unique functions we can create. This is the size of the search space. If a problem requires two functions of size $N$ (one function calling the other), without function composition we may need up to $2 \mathrm{~N}$ statements to solve this problem. That increases the search space to $E^{2 N}$ possibilities. In contrast, if we have an additional function, we have $E+1$ expressions in the ExpStore. Therefore, the number of unique SolFuncs we can create becomes $(E+1)^{N}$. For non-trivial cases,

$$
E^{2 N}>>(E+1)^{N} \text {. }
$$

Therefore, function composition is much more effective at reducing search space than allowing more statements in SolFunc.

Although genetic mutation and recombination get the most attention, composition can be also seen in biological cell evolution, where it is called endosymbiosis [42, p. 77]. For instance, it is widely believed that mitochondria present in eukaryotic cells (like animal or plant cells) have been captured from the primitive environment, where mitochondria existed independently as a more primitive prokaryotic cell (like bacteria). However, for mitochondria to evolve as a prokaryot, it must have solved some natural challenge (problem). In fact, it solved the problem of energy production on its own and is the 'power plant' in a eukaryotic cell. This shows that having many problems to solve is a key to evolution. This is the main insight used by AAD; if we want to solve larger problems, there should be many other simpler problems present, to guide evolution.

\subsubsection{Ganged Evolution}

Related problems, which have the same number and types of inputs and outputs (e.g., Min and Max, or SortAsc and

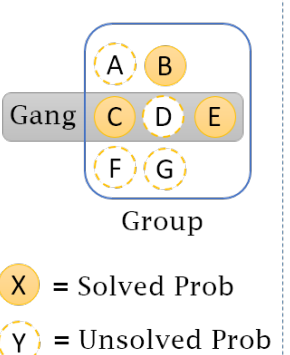

(a)

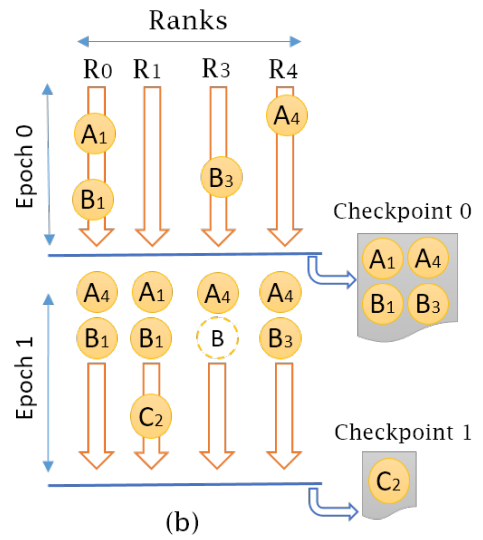

Figure 4. (a) How problems are grouped into gangs and groups. (b) Distribution of solutions via process joins and checkpoints. A, B, C are problems, with the subscript indicating the rank on which the solution is found.

SortDesc), are ganged together into a single gang (see Figure 4(a)). Once a SolFunc is generated for one of the problems in the gang, at the end of Phase 2 or 3, it is tested on all problems in the gang. This allows solutions to be found faster because a potential solution (SolFunc) may satisfy one of the many problems in the gang.

\subsubsection{Solution Mutation}

Instead of building a SolFunc from scratch in Phase 1, sometimes the Evolver picks an existing solution for another problem in the same gang, and attempts to mutate it by sending it through Phases 1, 2 and 3. In AAD, solution mutation is a trade-off, because if we pick an already built solution for mutation, we lose the opportunity to build a fresh SolFunc in that step. Notice that solution-mutation is different from operator-mutation described under Phase 3.

Natural evolution also takes advantage of multiple, related problems present in an environment to test new solutions or adapt existing ones for new purposes, as in the case of evolution of birds' feathers, which are believed to have first served a thermoregulatory or a display function [42, p. 671].

\subsubsection{Cross-Pollination Among Ranks}

AAD creates multiple concurrent processes (called ranks) and assigns problems to each of them to solve. Evolution happens in multiple ranks in isolation, in periods called epochs, as shown in Figure 4(b). Pseudo-code for the high-level evolution algorithm for one epoch is shown in Figure 5.

At the end of an epoch, synchronization happens and solutions are exchanged among ranks, as shown in Figure 4(b). At the end of an epoch, solutions discovered by each rank are sent to the master rank, which collects and distributes all of them to all ranks to be used in the next epoch. In the 


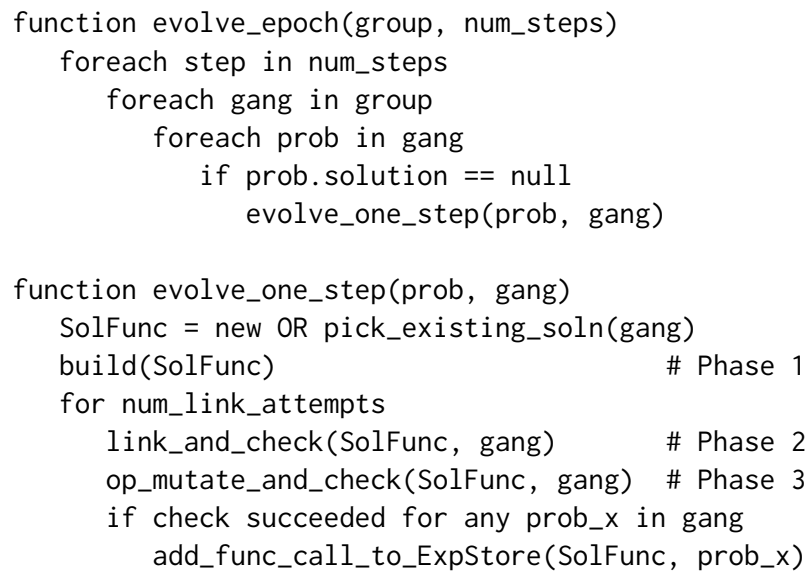

Figure 5. Evolution algorithm for single epoch.

next epoch, a rank may receive solutions discovered by any rank in the previous epoch (see Figure 4(b)).

\subsubsection{Diverse Environments \& Solutions}

In $\mathrm{AAD}$, each rank maintains its own copy of ExpStore and AAD allows some of the non-essential expressions in Table 1 to be randomly removed form a rank's ExpStore. In current setup, binary Arithmetic operations are added $80 \%$ of the time to a rank, Pop at Ind expressions are added $20 \%$ of the time, and Reduction idiom is added $10 \%$ of the time. Further, when a For Stmt is inserted, $20 \%$ of the time we use an enumerated For Stmt instead of a non-enumerated one. Moreover, when a rank receives a solution to a problem, a function call for it is added to ExpStore only $80 \%$ of the time. These random omissions of expressions, including function calls, help create diverse environments with respect to expressions available in the ExpStore.

Additionally, AAD allows multiple solutions (e.g., 100) to coexist for a given problem. For a solved problem, a rank may receive any one of these existing solutions, picked at random. This allows solution mutation to start from different solutions. Further, currently, $20 \%$ of the ranks do not receive a solution for a given problem, even when one exists, forcing them to find their own solutions. This is illustrated in Figure 4(b) with an empty circle, where Rank R3 does not receive a solution for problem $B$. Both of these strategies increase diversity of solutions.

These strategies are inspired by biological evolution, which uses diverse environments resulting from different temperatures, salinity, humidity, pressure, etc., to come up with different organisms. Similarly, evolution depends on diversity of individuals (solutions) within a population.

\subsection{Engineering Challenges}

Since AAD supports loops and conditional statements and uses function composition, $\mathrm{AAD}$ presents several challenges that are not faced by simpler program synthesizers. We take a practical engineering approach to solving these challenges as outlined below.

\subsubsection{Exceptions}

Although AAD produces syntactically correct programs, many runtime exceptions are possible due to various reasons ranging from divide by zero to popping from an out-of-bound index. Fortunately, Python provides a robust exception handling framework and $\mathrm{AAD}$ catches all exceptions arising from Python's built-in exec() function used to execute SolFunc.

Allowing exceptions in the first place does not make AAD less robust. As an engineering example, out-of-order processors introduce unintended exceptions such as page faults, divide by zero exceptions, through speculative actions taken by the processor itself $[8,11,52]$. However, processors have mechanisms to detect such violations and correct themselves, thereby making them robust.

\subsubsection{Infinite Loops}

Even natural evolution may cause infinite repetition, as seen with cancerous cell division. Instead of trying to detect infinite repetion (loops), we use a timeout to terminate programs that do not terminate within a given time period (e.g., 1 second), using Python's signal module to set an ALRM signal. Although it may appear as a crude solution, this is a well established engineering technique used in complex systems like microprocessors and spacecrafts, which use various watchdog timers $[29,53,54]$ to recover from a multitude of unpredictable situations like deadlocks, live-locks (starvation), soft errors, etc. For instance, when an execution core of a microprocessor issues a memory read request, if it gets dropped in the memory system due to an unexpected condition, the core may timeout and reissue the request.

However, there is a cost to this approach: timeouts waste valuable processing time. Therefore, we use one heuristic to prevent one such cause of infinite loops. When we iterate over an array $A$ using a for loop, allowing $A$ to grow by appending more items can cause an infinite loop. In Python, this can be easily prevented by making $A$ a tuple, which is immutable.

\subsubsection{Infinite Recursion}

Since cycles in call graph can lead to infinite recursion, AAD disallows any recursion. Since recursion detection is well understood $[7,14]$, AAD detects and discards programs with recursion. When we construct SolFunc, we do not let SolFunc call itself. When we call an already built function $F$ within SolFunc, we do not allow $F$ to call SolFunc either. Therefore, we do not have a cycle between SolFunc and $F$. We repeat this cycle detection process for any function $F$ we use. 


\subsection{Parallelization and HPC}

The parallelization of the evolutionary search process naturally lends to High-performance Computing (HPC), where a large number of processors and nodes are used to solve a problem. Therefore, we designed $\mathrm{AAD}$ as a multiprocess application, using Python's multiprocessor module, to take advantage of multiple processors on a single node. In addition, as shown in Figure 4(b), AAD can take a checkpoint of solutions discovered since the last checkpoint. With distributed file systems available on HPC clusters, this allows solutions found on node $N$ to be exchanged with other nodes, by those nodes reading the checkpoint dumped by node $N$. A node can read any available checkpoint at the start of an epoch, and does not wait for any specific checkpoint, thereby avoiding any synchronization. A checkpoint is produced using Python's pickle module.

However, parallel processing introduces other engineering challenges, including load balancing. Due to diverse environments and random nature of evolutionary decisions, some ranks may take longer to finish an epoch than others. Similarly, a large number of timeouts due to infinite loops can also increase the execution time of a rank. To mitigate the latter problem, a rank counts the number of total timeouts and if it is greater than a threshold, ends epoch early.

Strategies like discarding of solutions and early termination of search are made possible by the non-deterministic evolutionary nature of AAD. Evolution does not put much value on a single individual or even a single environment, but mainly depends on the continuation of the process itself. As long as evolution continues, a solution may be found one way or the other.

\section{Methodology}

The entire AAD framework is written in Python and is about 6,700 lines of code (see Appendix E), including all problem generators and checkers, blank lines and comments.

We use 3 groups of 10 problems each (described in Appen$\operatorname{dix}$ A) to study the effectiveness of evolutionary strategies described in Section 2.5. All problems are listed in Section 4.1.1. GroupA consists of typical list manipulation problems (e.g., min, sort, reverse, index, remove), GroupB represents basic vector processing problems (e.g., dot-product, matrix-vector multiply), and GroupC consists of some basic spreadsheet problems (e.g., sum, sum-if, count-if, average). One entire group is evolved in a given run. We run 112 concurrent processes on a 4-socket Intel Xeon(R) 8180 Platinum server with a total of 112 physical cores. To minimize artifacts due to randomness, we do 10 runs per experiment, for each group.

The metric used to evaluate different strategies is steps. Within a single step, we can send a SolFunc through phases 2 and 3 any number of times, to re-link and re-mutate. Currently, we do this twice, leading to 4 different variants. Since we simulate 112 ranks concurrently, each step reported accounts for $448(112 * 4)$ distinct SolFuncs. On the above server, on average, one step on a rank takes about $7 \mathrm{~ms}$ per gang. Runtime, however, is not an objective of this paper and we make no claims regarding runtime nor make any attempts to compare runtime of AAD to prior work.

For each problem, when a solution is found on a rank, it is reported by writing the solution and relevant statistics to a log file created for each rank. The first solution to be found for a problem among any rank is found through postprocessing log files and its step count is reported as the time to solution for that problem for that run. For an experiment with 10 runs, for a given problem, we report the average of all such step counts across all 10 runs.

For each run, we simulate a maximum of 100 epochs, with each epoch containing 2,000 steps. If at least one solution is found for all problems in a group before 100 epochs, we end the run and dump a checkpoint. For reporting purposes, AAD can rank solutions by reading checkpoints from one or more runs to find the least complex ones. Although there are many strategies to rank [18, 37, 44], we use a simple heuristic that assigns different weights to different structures - e.g., 50 for a function call, 20 for a For Stmt, 10 for an If Stmt, and 2 for any other statement. Ranking for all other purposes is left as future work.

AAD supports different parameters. Currently, we allow a maximum of 12 statements for a SolFunc, with additional 2 statements if SolFunc is mutated from an existing solution. We allow up to 100 different solutions to be found for a single problem. Default parameters used to create diverse environments were given in Section 2.5.5. All values were picked as reasonable guesses and tuning them is left as a future study.

There are no restrictions placed on inputs of problems other than all arrays must be non-empty. Problem generators usually generate input arrays from size 1 to 200, randomly filled with integers from -200 to +200 .

\section{Results and Analysis}

In this section, we present results showing the effectiveness of different evolutionary strategies discussed in Section 2.5, along with insights from code generated by AAD.

\subsection{Evolutionary Strategies}

\subsubsection{Composition}

Table 2 shows caller-callee relationships for GroupA problems (see Appendix B for all groups). For instance, the row for SortDesc function shows that SortAsc called Max in $57 \%$ of the solutions, Min in $14 \%$ of the solutions, and so on. Functions Min, Max, and ReverseArr did not call any other function. All other functions depended on one or more functions to arrive at a solution, underscoring the importance of 


\begin{tabular}{|c|c|c|c|c|c|c|c|c|c|c|}
\hline & 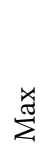 & $\Xi$ & 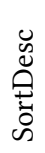 & 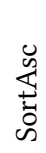 & 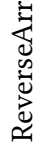 & 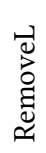 & 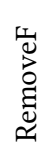 & 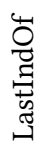 & 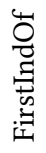 & 焉 \\
\hline \multicolumn{11}{|l|}{ Max } \\
\hline \multicolumn{11}{|l|}{ Min } \\
\hline SortDesc & 57 & 14 & & 43 & 43 & 14 & 57 & & & \\
\hline SortAsc & 4 & 8 & 94 & & 94 & 6 & 8 & & & 18 \\
\hline ReverseArr & & & & & & & & & & \\
\hline RemoveL & 2 & 1 & & & 26 & & 22 & 79 & & 10 \\
\hline RemoveF & 1 & 1 & & & 59 & 61 & & & 43 & 14 \\
\hline LastIndOf & & 2 & & & & & & & & \\
\hline FirstIndOf & & & & & & & 2 & & & \\
\hline IsInArr & 2 & 3 & & & 3 & 1 & 18 & 61 & 3 & \\
\hline
\end{tabular}

Table 2. Caller-callee relationships showing function composition for GroupA problems.

\begin{tabular}{|c|c|c|c|c|c|}
\hline GroupA & Baseline & Exp1 & Exp2 & Exp3 & Exp4 \\
\hline $\operatorname{Max}$ & 98 & 0.9 & 1.1 & 0.9 & 1.1 \\
\hline Min & 90 & 0.8 & 2.6 & 0.9 & 1.0 \\
\hline SortDesc & 59929 & $\infty$ & $\infty$ & $\infty$ & 1.2 \\
\hline SortAsc & 60177 & $\infty$ & $\infty$ & $\infty$ & 1.2 \\
\hline ReverseArr & 160 & 0.9 & 1185.9 & 1.2 & 1.5 \\
\hline RemoveL & 2599 & $\infty$ & $\infty$ & 3.1 & 0.8 \\
\hline RemoveF & 4050 & $\infty$ & $\infty$ & 5.2 & 0.8 \\
\hline LastIndOf & 511 & 1.3 & $\infty$ & 0.7 & 2.1 \\
\hline FirstIndOf & 17077 & 1.8 & $\infty$ & 2.5 & 0.7 \\
\hline IsInArr & 269 & 0.8 & $\infty$ & 0.9 & 1.4 \\
\hline \multicolumn{6}{|l|}{ GroupB } \\
\hline AddArrays & 2349 & 1.0 & 0.7 & 2.7 & 1.3 \\
\hline MultArrays & 18797 & 0.3 & 10.6 & 7.5 & 3.1 \\
\hline Sum & 9 & 2.6 & 0.9 & 1.1 & 1.1 \\
\hline SumOfSq & 9373 & 7.9 & $\infty$ & 11.0 & 1.8 \\
\hline DotProd & 18290 & $\infty$ & $\infty$ & 7.8 & 2.9 \\
\hline MatVecMult & 54476 & $\infty$ & $\infty$ & $\infty$ & 2.1 \\
\hline AddToArr & 232 & 0.7 & 0.8 & 1.8 & 1.8 \\
\hline SubFromArr & 2995 & 1.9 & $\infty$ & 4.5 & 0.7 \\
\hline ScaleArr & 3682 & 0.5 & $\infty$ & 2.7 & 0.7 \\
\hline ScaledSum & 29 & 269.0 & $\infty$ & 2.0 & 0.8 \\
\hline \multicolumn{6}{|l|}{ GroupC } \\
\hline CountEQ & 91345 & $\infty$ & $\infty$ & 1.5 & 1.9 \\
\hline CountLT & 109444 & $\infty$ & 1.2 & 1.3 & 1.6 \\
\hline CountGT & 109431 & $\infty$ & 1.2 & 1.3 & 1.6 \\
\hline SumIfLT & 25109 & 5.7 & 5.7 & 5.4 & 1.7 \\
\hline SumIfGT & 25065 & 5.7 & 5.7 & 4.6 & 1.7 \\
\hline SumIfEQ & 25001 & 5.7 & 8.0 & 4.5 & 1.7 \\
\hline ScaledAvg & 7548 & $\infty$ & $\infty$ & 1.1 & 0.8 \\
\hline Sum & 7 & 18.6 & 0.6 & 1.6 & 2.3 \\
\hline Len & 28 & 0.5 & 1.1 & 0.5 & 0.2 \\
\hline Avg & 891 & $\infty$ & $\infty$ & 1.2 & 1.2 \\
\hline
\end{tabular}

Table 3. Effectiveness of different evolutionary strategies. Exp1: Composition, Exp2: Operator mutation, Exp3: Crosspollination among ranks, Exp4: Diversity of solutions.

function composition. Some of the function dependencies may be unexpected as discussed in Section 4.2.1.
Table 3 shows all three groups of problems, their baseline performance in terms of the step count, and compares the effectiveness of four evolutionary strategies against the baseline. For the four experiments shown, each entry gives the ratio between the number of steps taken without that strategy and that of the baseline. Baseline step counts span a wide range due to the varying complexity of problems. Since step counts for each entry is taken by averaging across multiple runs, when a run does not produce a solution for a given problem, the maximum step count $(200,000)$ is used for that run [31]. If none of the runs for a given experiment produces a solution for a given problem, symbol $\infty$ is used to indicate that fact.

Step counts for Exp1 are obtained by disabling the addition of function calls to ExpStore for solutions found (i.e., disabling composition). This increases time to solution by a significant factor for some problems (e.g., SumOfSq, ScaledSum) and makes it impossible to find any solutions for others (e.g., SortAsc, DotProd), underscoring the importance of composition, especially for more complex problems. However, not adding function calls to ExpStore shrinks the search space and hence can speed up solutions of some simpler problems (e.g, Len, IsInArr) that do not have to depend on other functions.

\subsubsection{Operator Mutation}

Exp2 disables operator-mutation by disabling Phase 3, leading to increased solution times and altogether unsolved problems, especially in GroupA. Solutions that depend on an alternative operation (e.g., multiply instead of addition) are susceptible to eliminating operator-mutation. However, having fewer (or late) solutions reduces search space by reducing function composition and hence can speed up solutions to problems that do not depend on operator-mutation (e.g., Sum).

\subsubsection{Cross-Pollination}

Exp3 in Table 3 disables cross-pollination among ranks by running a single epoch of 200,000 steps, thereby avoiding any exchange of solutions among ranks. Many problems that depend on others (e.g., SortAsc, SortDesc) are severely affected by lack of cross-pollination.

\subsubsection{Diversity}

Exp4 reduces the number of solutions maintained for a given problem from 100 to 1 , thereby decreasing the diversity of solutions for a given problem. This negatively affects several problems (e.g., Multarrays, DotProd) demonstrating the importance of solution-diversity. Similarly, for GroupC, Exp5 in Table 5 shows the effect of adding all optional expressions described in Section 2.5.5 100\% of the time, decreasing diversity of environments and increasing expressions available to every rank. This shows that for many problems in GroupC adding optional expressions randomly is a better choice than 


\begin{tabular}{|c|c|c|c|c|c|c|c|c|c|c|}
\hline & $\begin{array}{l}\text { Ol } \\
\text { İ } \\
\vdots \\
0 \\
0\end{array}$ & 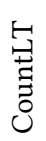 & 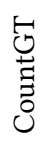 & 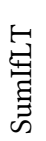 & 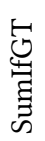 & 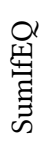 & 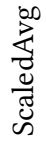 & 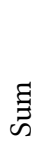 & ฮ્త્త & $\sum^{\infty}$ \\
\hline CountEQ & & 38 & 33 & 1 & 6 & 19 & & & & \\
\hline CountLT & 43 & & 33 & 2 & 3 & 16 & & & & \\
\hline CountGT & 43 & 36 & & 1 & 3 & 15 & & & & \\
\hline SumIfLT & 2 & & & & 41 & 56 & & & & \\
\hline SumIfGT & 1 & 1 & & 43 & & 53 & 1 & & & \\
\hline SumIfEQ & & 1 & & 42 & 53 & & 1 & & & \\
\hline ScaledAvg & & & & 22 & 25 & 34 & & & & \\
\hline Sum & & & & & & & & & & \\
\hline Len & & & & & & & & 5 & & \\
\hline Avg & & & & & & & & & & \\
\hline
\end{tabular}

Table 4. $\%$ of solutions where a solution mutated from another solution (a parent), for GroupC problems.

\begin{tabular}{|c|c|c|c|c|c|c|c|c|c|c|}
\hline & 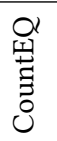 & 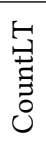 & 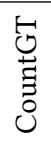 & 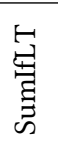 & 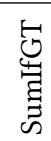 & 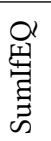 & 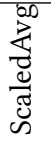 & $\begin{array}{c}\Xi \\
\Xi \\
\omega\end{array}$ & 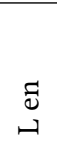 & ${ }^{\infty}$ \\
\hline Exp5 & 1.8 & 1.4 & 1.4 & 2.0 & 2.0 & 2.0 & 2.1 & 1.3 & 0.4 & 1.5 \\
\hline Exp6 & 2.0 & 1.6 & 1.6 & 3.7 & 5.8 & 2.2 & 0.9 & 0.6 & 0.4 & 0.9 \\
\hline
\end{tabular}

Table 5. Effectiveness of diversity of environments (Exp5), solution mutation (Exp6).

\begin{tabular}{|l|cc|c|}
\hline & AddArrays & MultArrays & Total \\
\hline Separate & 2023 & 7866 & 9889 \\
Together & 1746 & 6105 & 6105 \\
\hline
\end{tabular}

Table 6. Exp7: Effectiveness of ganged-evolution.

always adding them to every rank. However, as before, simpler problems (e.g., Len) can benefit from the late discovery of other solutions.

\subsubsection{Solution Mutation}

Table 4 gives $\%$ of solutions where a solution is mutated from another solution (a parent) for GroupC problems, where this is most common (see Appendix C for all groups). It should be noted that mutation can happen in either direction, on different ranks. For instance, some ranks may first come up with Min (or receive Min as an already solved problem from another rank) and Max may mutate out of it. On other ranks, Min may mutate out of Max.

Exp6 in Table 5 shows the effects of disabling solutionmutation for GroupC problems, which are dependent on parents as given by Table 4 . Problems that mutate from parents show increased time to solution, while problems that do not (e.g., Len) see a speedup, as in previous experiments.

\subsubsection{Ganged Evolution}

To show the effectiveness of ganged-evolution, we picked two problems that belong to the same gang, evolved them one at a time, and compared the results to evolving them together, as shown with Exp7 in Table 6. To isolate effects of ganged-evolution, we disabled function composition and solution-mutation, and picked two problems that can evolve

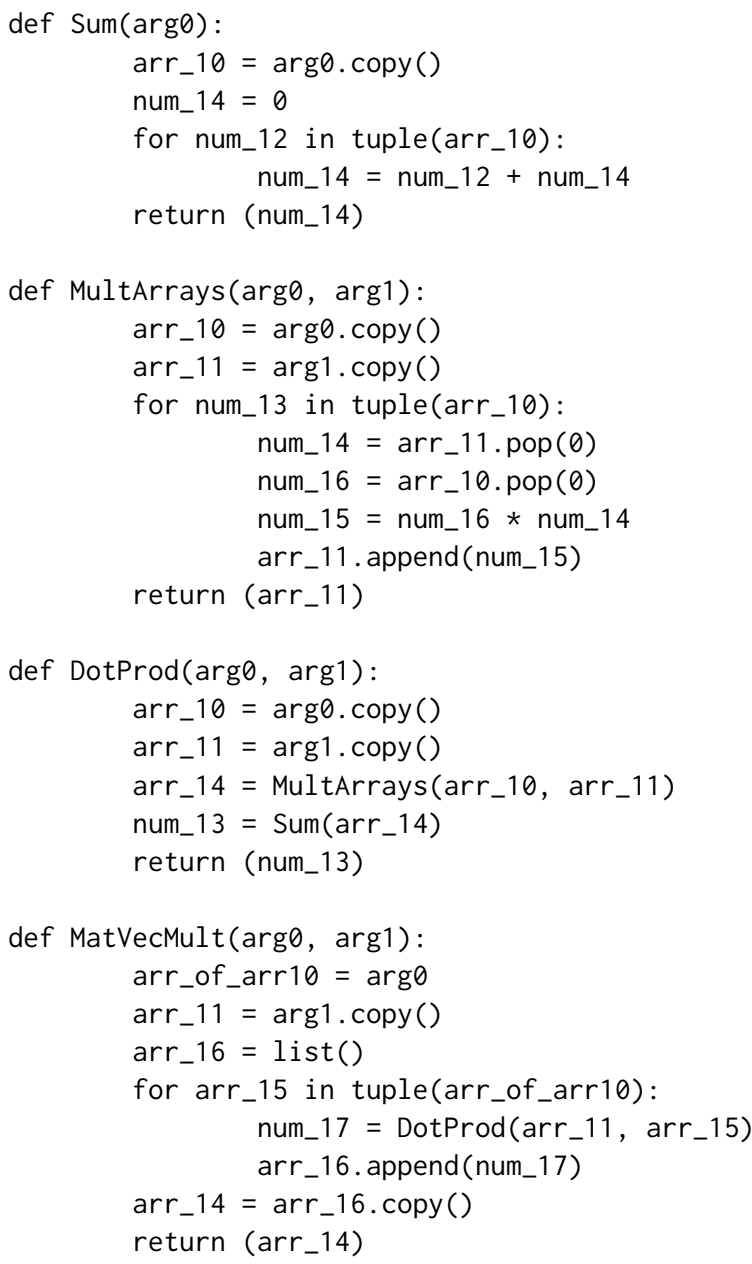

Figure 6. Code for MatVecMult

without other functions. Results show that evolving both of them separately takes about $1.6 \mathrm{X}$ steps $(9,889)$ compared to evolving them together $(6,105)$. Total column reports total steps to find solutions for both problems, in each case.

It should be emphasized that every evolutionary strategy is not important for every problem. Some simple problems can directly evolve from the grammar itself and they are often hurt by advanced strategies used. However, as these results show, many complex problems cannot find a solution without these strategies, within a reasonable time limit.

\subsection{Code Examples and Insights}

Being able to generate complex code is an important result of AAD. Besides SortAsc (from GroupA) shown in Section 1, in this section we show an example each from GroupB and GroupC. Code generated for all 29 problems is shown in Appendix D. Since AAD finds multiple solutions for a problem, we only discuss one of them, usually the least complex one.

The solution for MatVecMult (Figure 6) is performing dot products (DotProd) between row vectors in the matrix $(\arg 0)$ 
and the input vector ( $\arg 1)$, and appending those results to a new result vector. DotProd in turn depends on the sum (Sum) of two arrays multiplied together (MultArrays). MatVecMult is performing a linear transformation, which is the basis of linear algebra, and hence this discovery of AAD is particularly noteworthy.

From GroupC, the solution for CountEQ, which counts the number of times an element occurs in an array, is shown below. The algorithm is somewhat circuitous, because first it appends matching elements to a new list and then finds the length of that list using Len. This is an example of a non-obvious algorithm, although it is not the most efficient solution.

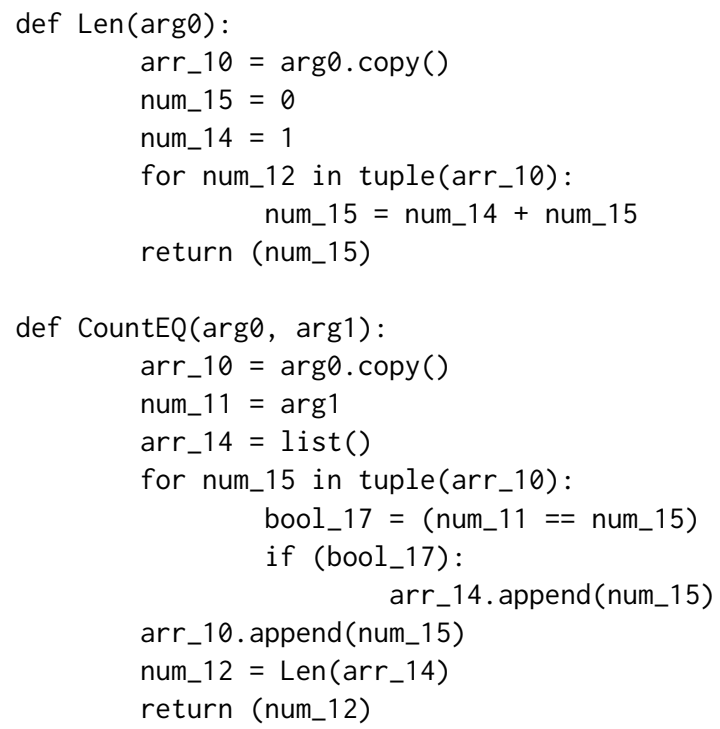

It should be noted that the code generated by AAD often contains redundant operations like copying, appending and popping the same element, calling functions whose results are never used, if-statements with always True or False conditions, etc. Some of these can be easily eliminated with standard compiler techniques and are out of scope for the current version of AAD.

\subsubsection{Outside-of-the-box Solutions}

This section describes some unexpected programs illustrating both strengths and weaknesses of AAD. Since AAD uses a limited number of input combinations generated by a ProbGen to check the validity of a program, any solution generated for a problem is as good as the ProbGen and the Checker used. This is both a weakness and a strength depending on the application. If an application demands rigorous validation, it is the responsibility of the ProbGen and the Checker to cover all cases, including corner cases. Writing such verification logic can be quite demanding, which is an obvious weakness. On the other hand, when an application needs to take advantage of peculiarities of the input, or needs to come up with solutions in constrained environments, AAD can show remarkable adaptability.
As a simple example, the grammar we use does not have truth values True and False provided as constants. Although initially this was an omission on our part, we realized that $\mathrm{AAD}$ was actually generating these values when they were needed, using an expression of the form bool_10 = (num_11 $==$ num_11). Similarly, initially, we forgot to include constant values 0 and 1 in the grammar. AAD overcame that difficulty by subtracting the same value from itself to generate zero and dividing the same value (e.g., the last value of an array) by itself to to generate constant 1 . Although, the latter approach is not safe because the last value of an array could be zero, it used that in cases where the ProbGen did not put a zero at the end of an array. To defeat this, we changed ProbGen to generate an array of all zeros for some problem sizes. Then AAD generated constant value 1 by taking advantage of Len - by dividing the length of the array by itself, because we always use non-empty arrays. This shows that the SolGen is in an adversarial relationship with ProbGen/Checker, trying to defeat the latter duo by exploiting any opportunity or weakness present, similar to bacteria adapting to anti-biotics in biolgical evolution.

Another such example is FirstInd0f, which is used for finding the index of a given element in an array. If there are multiple elements present, the function returns the index of the first element found. Most programmers would write a for-loop to iterate over the array looking for the element, and when a match is found, will break out of the loop using a break or a return statement. However, the current grammar used by AAD does not have a break statement and does not use return statements in the middle of a function. One solution AAD came up with to solve this challenge is given below. First, it goes through the array popping each element from the front. If a match is found, it is appended at the end of the same array. Once it has gone through the loop, all the elements left in the array are the matching elements appended at the end. Then this function returns the head of the remaining array, which is the first matching index. In other situations, we have also seen it calling Min function to find the minimum value of the remaining array.

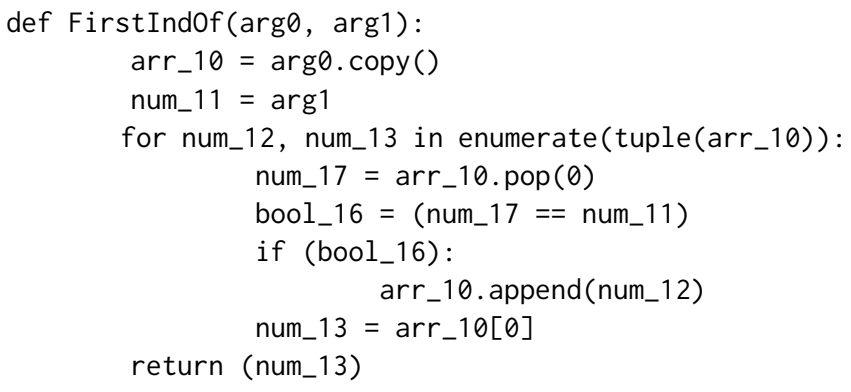

FirstIndOf is usually defined with a pre-condition - viz., the element must be always present (e.g., index () in Python). With our ProbGen/Checker, the behavior of this function is not defined if arg1 is not present in the array. We observed Is InArr function exploiting this undefined return value of a 
specific FirstIndOf function to arrive at a solution, which always passed our checker but was incorrect in a rare situation the Checker did not check for. Although such a solution is problematic in many uses, it illustrates the ability to take advantage of deficiencies (or features) present in the inputs or the Checker. This could be quite valuable for an autonomous system to adapt to (or take advantage of) vulnerabilities of an adversary.

The following solution for IsInArr is a prime example of outside-of-the-box thinking. This code finds whether a given element $(\arg 1)$ is in a given array $(\arg 0)$. Most programmers would write a loop that goes over the array and looks for a match. However, the following program does not contain any loops, which appeared to be a bug until we realized what it was doing. It first appends arg1 to the end of the array. Then, it calls RemoveF to remove arg1, which removes the first matching element. Then it pops the last element of the array and checks whether it matches arg 1 it appended. If it matches arg1, removeF must have removed another element equal to arg1 from the array. In that case, there must have been another element already present in the array and IsInArr must return true, as it does here. If the popped element does not match arg1, it means RemoveF removed arg1 it appended itself. In that case, there was no prior matching element and IsInArr must return False, as is the case.

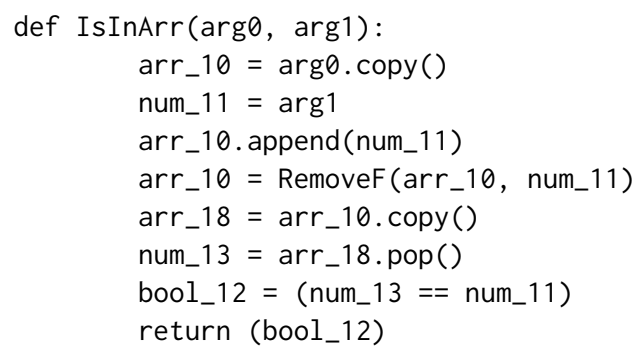

If any person were to come up with this solution for IsInArr, we would have labeled him or her as creative or an outside-of-the-box thinker. It is hard to believe that a machine is capable of this level of logical reasoning, although AAD stumbled upon it without any reasoning at all. This capability presents us with a new opportunity for AAD - viz. to use it as an outside-of-the-box thinker to come up with alternative solutions that we would not normally think of. After all, creative thinking does not seem to be the prerogative of humans alone.

\section{Related Work}

Program synthesis is an active and challenging research area that seeks to automate programming. Many approaches have been proposed over the years in an effort to generate programs that conform as accurately as possible to the userexpressed intent. In [17] Gulwani, Polozov, and Singh present an excellent survey on the program synthesis problem, applications, principles and proposed solutions. In [40], the same authors extend their prior survey to include more recent advances that span 2017 and 2018. Below, we review related work in the area, discuss typical problem domains, challenges in program synthesis and position our work among prior research.

Program synthesis approaches have targeted automatic generation of solutions for problems in domains such as data wrangling $[15,30,43,45]$, graphics [10, 19], code repair [22, 35, 46], superoptimization [4, 24, 33, 38], and others. In the above cases solutions are sought for restricted target problem domains such as string manipulation [15], bit-vector programs [21], optimized code implementations at the ISA level [38], etc. Moreover, many program synthesis approaches are restricted to straight-line code fragments $[3,4$, 38]. While there exist works that target loop-based code, they are either restricted in the form of SIMD code [5] or synthesis of loop bodies (e.g., within templates [49] or sketches [48]); the majority of related work, unlike $\mathrm{AAD}$, focus on loopfree programs [16, 21]. However, program synthesis tools like SYNQUID [39], MYTH [36], and Leon [26] can generate recursive and/or higher-order programs that can be as expressive as loop-enabled approaches, in frameworks with formal specifications. AAD enables similar capabilities for general-purpose Python language with its support of loops, function composition, and complex control flow.

Two of the main challenges in program synthesis are related to the intractability of program search space and accurately expressing user intent. There are many search techniques proposed to address the former problem: enumerative, deduction-based [41], constraint solving [47, 49], as well as stochastic search techniques [28, 34]. Stochastic search techniques include a lot of modern approaches that employ machine learning $[32,34]$ and neural program synthesis $[3,12,51,55]$. In comparison, AAD uses a modified evolutionary approach that relies on PGE without requiring a fitness function.

As far as expressing user intent is concerned, different program synthesis techniques use formal logical specifications (mostly deductive-based techniques), informal natural language descriptions, and templates and sketches, among others. In $\mathrm{AAD}$ we provide the specification in the form of a program (called a checker) along with test inputs. This is similar to oracle-guided synthesis [21] (where an oracle produces the correct output) and reference implementations in SKETCH [47].

To show the effectiveness of AAD, we use array based problems similar to the benchmarks (e.g., array-search) in the SyGuS-Comp competition [1]. However, solvers such as Sketch-based, enumerative or stochastic, do not scale up to large array sizes [2], although it may be conceptually possible to extend Sketch based templates to express the grammar we use, in order to support large arrays. AADâĂŹs support of loops allows it to support input arrays of any (non-zero) size, and hence be as effective as frameworks that support recursion and/or higher-order operators. 
Overall, our work complements and builds upon prior approaches that use composition; Bladek and Krawiec [6] propose a similar genetic programming approach of simultaneous synthesis of multiple functions, and briefly explain the concept with four simple examples (last, patch, splice, splitAt). AAD extends this to much more complex problems with PGE and associated evolutionary strategies. Although, other works, like SYNQUID [39] utilize components in the process of program synthesis, unlike the former, PGE does not require the user to specify any underlying order (dependence) between the constituent components/functions. Besides, not specifying dependencies allows AAD to discover outside-of-the-box solutions.

Compared to other works (e.g., deductive-based approaches), program equivalence in $\mathrm{AAD}$ is not formally proven. This is typical in similar approaches of counter-example guided synthesis where "the best validation oracle for most domains and specifications tends to be the user" [17], who can inspect the program under consideration. We also emphasize that formal verification is not a prerequisite for many useful applications, especially for knowledge discovery for AI (e.g., for a robot to find a way to sort objects for packing). Human knowledge in general is inductive in nature. After all, biological evolution produced complex and intelligent organisms as humans without anyone writing a formal specification.

\section{Discussion \& Future Work}

This section discusses limitations of AAD, alternative ways of guiding evolution, and potential applications of AAD.

\subsection{Limitations of AAD}

A large search space is a challenge to search-based synthesizers [17]. For AAD, this is especially true due to addition of function calls as new solutions are discovered. AAD depends on guiding to solve this challenge and Section 6.2 outlines several possible ways for guiding.

The problems solved in this paper mostly require regular control flow (except Firstindof and RemoveF). Programs that require complicated control-flow may take considerably more time to be discovered. Similarly, algorithms that depend on a very specific value (e.g., if $x<3.5$ ) are hard for AAD to discover, unless those values are present. $\mathrm{AAD}$ would be more suitable for performing permutations and combinations of already available inputs, with straightforward numerical processing. The solution for the above deficiency is to have proper library support. For instance, although it may be difficult for AAD to produce an algorithm for FFT (Fast Fourier Transform) on its own, it should be able to call an FFT implementation in a library and use that to solve other problems.

\subsection{Guiding the Hand of Evolution}

Grouping problems for PGE can be achived in several ways. First, we can imagine humans (domain experts) doing guiding. For instance, future 'programmers' or scientists could just suggest $A A D$ to use problems $A$ and $B$ to come up with a solution for problem C (e.g., "try using dot product to come up with an algorithm for matrix-vector multiply"). Notice that this is quite analogous to the way we teach children to discover solutions to problems on their own ("try using a screwdriver instead of a hammer"). Similarly, a researcher who wants to come up with a hypothesis, or a programmer who wants to come up with a heuristic, may be able to make some suggestions and let AAD discover an algorithm, especially a non-obvious one, based on that guidance. If the Checker is based on past data or sensor data from the physical environment, this strategy could be used on many real-world problems without having to write a Checker or a ProbGen as we discussed in Section 2.2. This would be an entirely new way to "program" computers and build scientific models, and we intend to pursue this further.

Second, we can imagine other AI programs doing this guiding, especially in restricted domains where AI systems can guess the components of a solution based on domain, but not the exact algorithm [3].

\subsection{Other Potential Applications}

Conceptually, AAD can also be used in program translation. If we have a routine written in $\mathrm{C}$, assembly, or even binary language, we can execute that routine as a Checker for AAD to produce code in Python (or similar). This is akin to a machine learning an algorithm by just observing how another one behaves (i.e., how another one responds to inputs). Incidently, Python code shown in this paper can be considered as Python to Python translations, since the Checker is a different Python implementation.

$A A D$ could be more than a program synthesizer. It could be used to acquire intrinsic knowledge for machines. The callercallee graph (Table 2) and the parent-child graph (Table 4) capture inherent relationships between different problems. For instance, we can see $\mathrm{min} / \mathrm{max}$ is related to sorting, and dot-product to matrix-vector multiply. These relationships are discovered by AAD itself and can be thought of as one representation of associative memory among actions, similar to what human brains construct (e.g., getting ready in the morning is associated with brushing teeth, dressing up, etc.). Since AAD allows incremental expansion of knowledge by introducing more and more problems, with a proper guiding mechanism we may be able to guide autonomous systems to acquire a large number of skills (algorithms) and build a knowledge representation on their own, the same way we guide our children to acquire a large body of skills and knowledge by presenting them with many problems and challenges over their childhood. 


\section{Conclusion}

We presented AAD, an evolutionary framework for synthesizing programs of high complexity. Using a basic subset of Python language as grammar, AAD allowed us to synthesize code for 29 array/vector problems, ranging from min, max, reverse to more challenging problems like sorting and matrix-vector multiplication, without input size restrictions. AAD's use of problem guided evolution (PGE) and related evolutionary strategies made this possible. We evaluated the effectiveness of these strategies and presented evidence of outside-of-the-box problem solving skills of AAD. To deal with various challenges posed by complex requirements, we demonstrated how to use HPC techniques. Overall, we show that evolutionary algorithms with PGE are capable of solving problems of similar or higher complexity compared to the state-of-the-art.

\section{References}

[1] [n. d.]. Syntax-Guided Synthesis Competition. http://www.sygus.org/.

[2] R. Alur, R. Bodik, G. Juniwal, M. M. K. Martin, M. Raghothaman, S. A. Seshia, R. Singh, A. Solar-Lezama, E. Torlak, and A. Udupa. 2013 Syntax-guided synthesis. In 2013 Formal Methods in Computer-Aided Design. 1-8.

[3] Matej Balog, Alexander L. Gaunt, Marc Brockschmidt, Sebastian Nowozin, and Daniel Tarlow. 2016. DeepCoder: Learning to Write Programs. CoRR abs/1611.01989 (2016). arXiv:1611.01989

[4] Sorav Bansal and Alex Aiken. 2008. Binary Translation Using Peephole Superoptimizers. In Proceedings of the 8th USENIX Conference on Operating Systems Design and Implementation (OSDI'08). USENIX Association, Berkeley, CA, USA, 177-192.

[5] Gilles Barthe, Juan Manuel Crespo, Sumit Gulwani, Cesar Kunz, and Mark Marron. 2013. From Relational Verification to SIMD Loop Synthesis. In Proceedings of the 18th ACM SIGPLAN Symposium on Principles and Practice of Parallel Programming (PPoPP '13). ACM, New York, NY, USA, 123-134.

[6] Iwo Bladek and Krzysztof Krawiec. 2016. Simultaneous Synthesis of Multiple Functions Using Genetic Programming with Scaffolding. In Proceedings of the 2016 on Genetic and Evolutionary Computation Conference Companion (GECCO '16 Companion). ACM, New York, NY, USA, 97-98. https://doi.org/10.1145/2908961.2908992

[7] Richard P Brent. 1980. An improved Monte Carlo factorization algorithm. BIT Numerical Mathematics 20, 2 (1980), 176-184.

[8] Roger A Bringmann, Scott A Mahlke, Richard E Hank, John C Gyllenhaal, and W Hwu Wen-mei. 1993. Speculative execution exception recovery using write-back suppression. In Microarchitecture, 1993., Proceedings of the 26th Annual International Symposium on. IEEE, 214223.

[9] A. Chaudhuri, K. Mandaviya, P. Badelia, and S.K. Ghosh. 2016. Optical Character Recognition Systems for Different Languages with Soft Computing. Springer International Publishing, 53.

[10] Ravi Chugh, Brian Hempel, Mitchell Spradlin, and Jacob Albers. 2016. Programmatic and Direct Manipulation, Together at Last. SIGPLAN Not. 51, 6 (June 2016), 341-354.

[11] Harry Dwyer and Hwa C Torng. 1992. An out-of-order superscalar processor with speculative execution and fast, precise interrupts. $A C M$ SIGMICRO Newsletter 23, 1-2 (1992), 272-281.

[12] Yu Feng, Ruben Martins, Osbert Bastani, and Isil Dillig. 2018. Program Synthesis Using Conflict-driven Learning. In Proceedings of the 39th ACM SIGPLAN Conference on Programming Language Design and Implementation (PLDI 2018). ACM, New York, NY, USA, 420-435.
[13] Jonathan Frankle, Peter-Michael Osera, David Walker, and Steve Zdancewic. 2016. Example-directed Synthesis: A Type-theoretic Interpretation. SIGPLAN Not. 51, 1 (Jan. 2016), 802-815. https: //doi.org/10.1145/2914770.2837629

[14] Peter Gammie. 2015. The Tortoise and Hare Algorithm. Archive of Formal Proofs (Nov. 2015). http://isa-afp.org/entries/TortoiseHare. html, Formal proof development.

[15] Sumit Gulwani. 2011. Automating String Processing in Spreadsheets Using Input-output Examples. In Proceedings of the 38th Annual ACM SIGPLAN-SIGACT Symposium on Principles of Programming Languages (POPL '11). ACM, New York, NY, USA, 317-330.

[16] Sumit Gulwani, Susmit Jha, Ashish Tiwari, and Ramarathnam Venkatesan. 2011. Synthesis of Loop-free Programs. SIGPLAN Not. 46, 6 (June 2011), 62-73.

[17] Sumit Gulwani, Oleksandr Polozov, and Rishabh Singh. 2017. Program Synthesis. Foundations and TrendsÂ̋̈ in Programming Languages 4, 1-2 (2017), 1-119.

[18] Tihomir Gvero, Viktor Kuncak, Ivan Kuraj, and Ruzica Piskac. 2013. Complete Completion Using Types and Weights. SIGPLAN Not. 48, 6 (June 2013), 27-38.

[19] Brian Hempel and Ravi Chugh. 2016. Semi-Automated SVG Programming via Direct Manipulation. In Proceedings of the 29th Annual Symposium on User Interface Software and Technology (UIST '16). ACM, New York, NY, USA, 379-390.

[20] V.G. Ivancevic and T.T. Ivancevic. 2007. Computational Mind: A Complex Dynamics Perspective. Springer Berlin Heidelberg, 243.

[21] Susmit Jha, Sumit Gulwani, Sanjit A. Seshia, and Ashish Tiwari. 2010. Oracle-guided Component-based Program Synthesis. In Proceedings of the 32Nd ACM/IEEE International Conference on Software Engineering Volume 1 (ICSE '10). ACM, New York, NY, USA, 215-224.

[22] Barbara Jobstmann, Andreas Griesmayer, and Roderick Bloem. 2005. Program Repair As a Game. In Proceedings of the 17th International Conference on Computer Aided Verification (CAV'05). Springer-Verlag, Berlin, Heidelberg, 226-238.

[23] Colin G. Johnson. 2007. Genetic Programming with Fitness Based on Model Checking. In Genetic Programming, Marc Ebner, Michael O’Neill, Anikó Ekárt, Leonardo Vanneschi, and Anna Isabel Esparcia-Alcázar (Eds.). Springer Berlin Heidelberg, Berlin, Heidelberg, 114-124.

[24] Rajeev Joshi, Greg Nelson, and Keith Randall. 2002. Denali: A Goaldirected Superoptimizer. SIGPLAN Not. 37, 5 (May 2002), 304-314.

[25] Gal Katz and Doron Peled. 2008. Genetic Programming and Model Checking: Synthesizing New Mutual Exclusion Algorithms. In Automated Technology for Verification and Analysis, Sungdeok (Steve) Cha, Jin-Young Choi, Moonzoo Kim, Insup Lee, and Mahesh Viswanathan (Eds.). Springer Berlin Heidelberg, Berlin, Heidelberg, 33-47.

[26] Etienne Kneuss, Ivan Kuraj, Viktor Kuncak, and Philippe Suter. 2013. Synthesis Modulo Recursive Functions. SIGPLAN Not. 48, 10 (Oct. 2013), 407-426. https://doi.org/10.1145/2544173.2509555

[27] John R. Koza. 1992. Genetic Programming: On the Programming of Computers by Means of Natural Selection. MIT Press, Cambridge, MA, USA.

[28] John R. Koza. 1994. Genetic programming as a means for programming computers by natural selection. Statistics and Computing 4, 2 (01 Jun 1994), 87-112.

[29] K. A. LaBel and M. M. Gates. 1996. Single-event-effect mitigation from a system perspective. IEEE Transactions on Nuclear Science 43, 2 (April 1996), 654-660.

[30] Vu Le and Sumit Gulwani. 2014. FlashExtract: A Framework for Data Extraction by Examples. In Proceedings of the 35th ACM SIGPLAN Conference on Programming Language Design and Implementation (PLDI '14). ACM, New York, NY, USA, 542-553.

[31] Woosuk Lee, Kihong Heo, Rajeev Alur, and Mayur Naik. 2018. Accelerating search-based program synthesis using learned probabilistic 
models. In Proceedings of the 39th ACM SIGPLAN Conference on Programming Language Design and Implementation. ACM, 436-449.

[32] Percy Liang, Michael I. Jordan, and Dan Klein. 2010. Learning Programs: A Hierarchical Bayesian Approach. In Proceedings of the 27th International Conference on International Conference on Machine Learning (ICML'10). Omnipress, USA, 639-646.

[33] Henry Massalin. 1987. Superoptimizer: A Look at the Smallest Program. In Proceedings of the Second International Conference on Architectual Support for Programming Languages and Operating Systems (ASPLOS II). IEEE Computer Society Press, Los Alamitos, CA, USA, 122-126.

[34] Aditya Krishna Menon, Omer Tamuz, Sumit Gulwani, Butler Lampson, and Adam Kalai. 2013. A Machine Learning Framework for Programming by Example, Vol. 28. Int'l Conf. Machine Learning, 187-195.

[35] H. D. T. Nguyen, D. Qi, A. Roychoudhury, and S. Chandra. 2013. SemFix: Program repair via semantic analysis. In 2013 35th International Conference on Software Engineering (ICSE). 772-781.

[36] Peter-Michael Osera and Steve Zdancewic. 2015. Type-and-exampledirected Program Synthesis. SIGPLAN Not. 50, 6 (June 2015), 619-630.

[37] Daniel Perelman, Sumit Gulwani, Thomas Ball, and Dan Grossman 2012. Type-directed Completion of Partial Expressions. SIGPLAN Not. 47, 6 (June 2012), 275-286.

[38] Phitchaya Mangpo Phothilimthana, Aditya Thakur, Rastislav Bodik, and Dinakar Dhurjati. 2016. Scaling Up Superoptimization. SIGPLAN Not. 51, 4 (March 2016), 297-310.

[39] Nadia Polikarpova, Ivan Kuraj, and Armando Solar-Lezama. 2016. Program Synthesis from Polymorphic Refinement Types. In Proceedings of the 37th ACM SIGPLAN Conference on Programming Language Design and Implementation (PLDI '16). ACM, New York, NY, USA, 522-538.

[40] Alex Polozov. 2018. Program Synthesis in 2017-18. https://alexpolozov. com/blog/program-synthesis-2018/.

[41] Oleksandr Polozov and Sumit Gulwani. 2015. FlashMeta: A Framework for Inductive Program Synthesis. In Proceedings of the 2015 ACM SIGPLAN International Conference on Object-Oriented Programming, Systems, Languages, and Applications (OOPSLA 2015). ACM, New York, NY, USA, 107-126.

[42] William K. Purves, David E Sadava, Gordon H. Orians, and H. Craig Heller. 2003. Life: the Science of Biology, 7th Edition. Sinauer Associates and W. H. Freeman.

[43] Rishabh Singh and Sumit Gulwani. 2012. Synthesizing Number Transformations from Input-output Examples. In Proceedings of the 24th International Conference on Computer Aided Verification (CAV'12). Springer-Verlag, Berlin, Heidelberg, 634-651.

[44] Rishabh Singh and Sumit Gulwani. 2015. Predicting a Correct Program in Programming by Example. In Computer Aided Verification, Daniel Kroening and Corina S. Păsăreanu (Eds.). Springer International Publishing, Cham, 398-414.

[45] Rishabh Singh and Sumit Gulwani. 2016. Transforming Spreadsheet Data Types Using Examples. SIGPLAN Not. 51, 1 (Jan. 2016), 343-356.

[46] Rishabh Singh, Sumit Gulwani, and Armando Solar-Lezama. 2013. Automated Feedback Generation for Introductory Programming Assignments. SIGPLAN Not. 48, 6 (June 2013), 15-26.

[47] Armando Solar-Lezama. 2008. Program Synthesis by Sketching. Ph.D. Dissertation. Berkeley, CA, USA. Advisor(s) Bodik, Rastislav. AAI3353225

[48] Armando Solar-Lezama, Gilad Arnold, Liviu Tancau, Rastislav Bodik, Vijay Saraswat, and Sanjit Seshia. 2007. Sketching Stencils. SIGPLAN Not. 42, 6 (June 2007), 167-178.

[49] Saurabh Srivastava, Sumit Gulwani, and Jeffrey S. Foster. 2013. Template-based program verification and program synthesis. International fournal on Software Tools for Technology Transfer 15, 5 (01 Oct 2013), 497-518.

[50] Emina Torlak and Rastislav Bodik. 2013. Growing Solver-aided Languages with Rosette. In Proceedings of the 2013 ACM International
Symposium on New Ideas, New Paradigms, and Reflections on Programming \& Software (Onward! 2013). ACM, New York, NY, USA, 135-152.

[51] Ashwin J. Vijayakumar, Abhishek Mohta, Oleksandr Polozov, Dhruv Batra, Prateek Jain, and Sumit Gulwani. 2018. Neural-Guided Deductive Search for Real-Time Program Synthesis from Examples. CoRR abs/1804.01186 (2018). arXiv:1804.01186

[52] David W Wall. 1994. Speculative execution and instruction-level parallelism. Report Western Research Laboratory (1994).

[53] N. J. Wang and S. J. Patel. 2006. ReStore: Symptom-Based Soft Error Detection in Microprocessors. IEEE Transactions on Dependable and Secure Computing 3, 3 (July 2006), 188-201.

[54] C. Weaver and T. Austin. 2001. A fault tolerant approach to microprocessor design. In 2001 International Conference on Dependable Systems and Networks. 411-420.

[55] Lisa Zhang, Gregory Rosenblatt, Ethan Fetaya, Renjie Liao, William E. Byrd, Raquel Urtasun, and Richard Zemel. 2018. Leveraging Constraint Logic Programming for Neural Guided Program Synthesis. https: //openreview.net/forum?id=HJIHtIJvz 


\section{Appendix A: Problem Definitions}

Table 1 lists all 3 groups of problems used in this work.

\begin{tabular}{|c|c|}
\hline Problem & Description \\
\hline \multicolumn{2}{|l|}{ GroupA } \\
\hline Max & $N U M=\operatorname{Max}(\mathrm{ARR})$. Returns maximum of an array \\
\hline Min & $N U M=\operatorname{Min}(\mathrm{ARR})$. Returns minimum of an array \\
\hline SortDesc & $\begin{array}{l}A R R=\text { Sor tDesc }(\mathrm{ARR}) \text {. Returns a sorted array in descend- } \\
\text { ing order }\end{array}$ \\
\hline SortAsc & $\begin{array}{l}A R R=\text { Sort Asc(ARR). Returns a sorted array in ascend- } \\
\text { ing order }\end{array}$ \\
\hline ReverseArr & $A R R=$ ReverseArr(ARR). Returns a reversed array \\
\hline RemoveL & $\begin{array}{l}\text { RemoveL(ARR, NUM). Removes the last occurrence of } \\
\text { given number }{ }^{*} \text { in array }\end{array}$ \\
\hline RemoveF & $\begin{array}{l}\text { RemoveF(ARR, NUM). Removes the first occurrence of } \\
\text { given number* in array }\end{array}$ \\
\hline LastIndOf & $\begin{array}{l}\text { NUM = LastIndOf(ARR, NUM). Returns the last index } \\
\text { of given number* in array }\end{array}$ \\
\hline FirstIndOf & $\begin{array}{l}N U M=\text { FirstIndOf(ARR, NUM). Returns the first index } \\
\text { of given number* in array }\end{array}$ \\
\hline IsInArr & $\begin{array}{l}B O O L=\text { IsInArr(ARR, NUM). Returns whether a given } \\
\text { number is in array }\end{array}$ \\
\hline \multicolumn{2}{|r|}{ ( } \\
\hline AddArrays & $\begin{array}{l}A R R=\text { AddArrays(ARR, ARR). Adds corresponding ele- } \\
\text { ments of two arrays together }\end{array}$ \\
\hline MultArrays & $\begin{array}{l}A R R=\text { Mult tarrays (ARR, ARR). Multiplies corresponding } \\
\text { elements of two arrays together }\end{array}$ \\
\hline Sum & $N U M=$ Sum(ARR). Returns sum of elements of an array \\
\hline SumOfSq & $\begin{array}{l}N U M=\text { SumOfSq(ARR). Returns sum of each element } \\
\text { squared in array }\end{array}$ \\
\hline DotProd & $\begin{array}{l}\text { NUM = DotProd(ARR, ARR). Returns dot product of two } \\
\text { arrays }\end{array}$ \\
\hline MatVecMult & $\begin{array}{l}A R R=\text { MatVecMult }(\mathrm{AoA}, \mathrm{ARR}) \text {. Returns result of a } \\
\text { matrix-vector multiply }\end{array}$ \\
\hline AddToArr & $\begin{array}{l}A R R=\text { AddToArr(ARR, NUM). Adds a number to each } \\
\text { element of an array }\end{array}$ \\
\hline SubFromArr & $\begin{array}{l}A R R=\text { SubFromArr(ARR, NUM). Subtracts a number } \\
\text { from each element of an array }\end{array}$ \\
\hline ScaleArr & $\begin{array}{l}A R R=\text { ScaleArr(ARR, NUM). Multiplies each element } \\
\text { of an array by a number }\end{array}$ \\
\hline ScaledSum & $\begin{array}{l}N U M=\text { ScaledSum(ARR, NUM). Multiplies each element } \\
\text { of an array by a number and sums the result }\end{array}$ \\
\hline \multicolumn{2}{|l|}{ GroupC } \\
\hline CountEQ & $\begin{array}{l}N U M=\text { CountEQ(ARR). Returns number of elements } \\
\text { equal to a given number in array }\end{array}$ \\
\hline CountLT & $\begin{array}{l}N U M=\text { CountLT(ARR). Returns number of elements less } \\
\text { than a given number in array }\end{array}$ \\
\hline CountGT & $\begin{array}{l}N U M=\text { CountGT(ARR). Returns number of elements } \\
\text { greater than a given number in array }\end{array}$ \\
\hline SumIfLT & $\begin{array}{l}N U M=\text { SumI } f E Q(A R R) \text {. Returns the sum of elements less } \\
\text { than a given number in array }\end{array}$ \\
\hline SumIfGT & $\begin{array}{l}\text { NUM = SumIfGT(ARR). Returns the sum of elements } \\
\text { greater than a given number in array }\end{array}$ \\
\hline SumIfEQ & $\begin{array}{l}N U M=\text { SumI } f E Q(A R R) . \text { Returns the sum of elements } \\
\text { equal to a given number in array }\end{array}$ \\
\hline ScaledAvg & $\begin{array}{l}\text { NUM = ScaledAvg(ARR). Returns the average of array } \\
\text { elements scaled by a number }\end{array}$ \\
\hline Sum & $N U M=$ Sum(ARR). Returns sum of elements of an array \\
\hline Len & $N U M=$ Len(ARR). Returns the length of array \\
\hline Avg & $N U M=\operatorname{Avg}($ ARR $)$. Returns the average of array \\
\hline
\end{tabular}

Table 1. GroupA, GroupB, and GroupC problems. Symbol * indicates that number must be present in array

\section{Appendix B: Composition Graphs}

Table 2 lists caller-callee relationships for all 3 groups of problems.

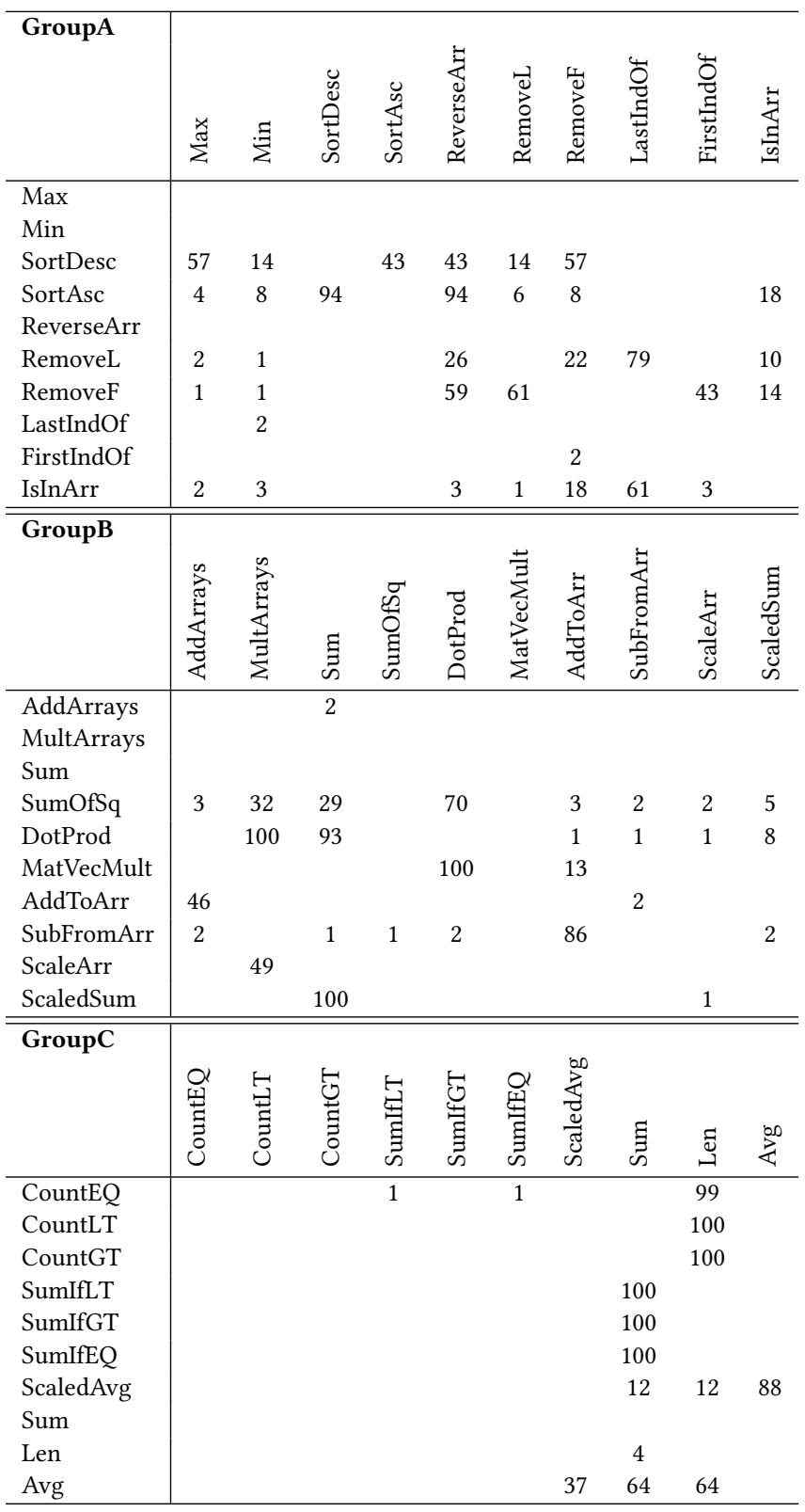

Table 2. Caller-callee relationships showing function composition for GroupA, GroupB, and GroupC problems. 


\section{Appendix C: Parent-Child Graphs}

Table 3 lists parent-child relationships for all 3 groups of problems.

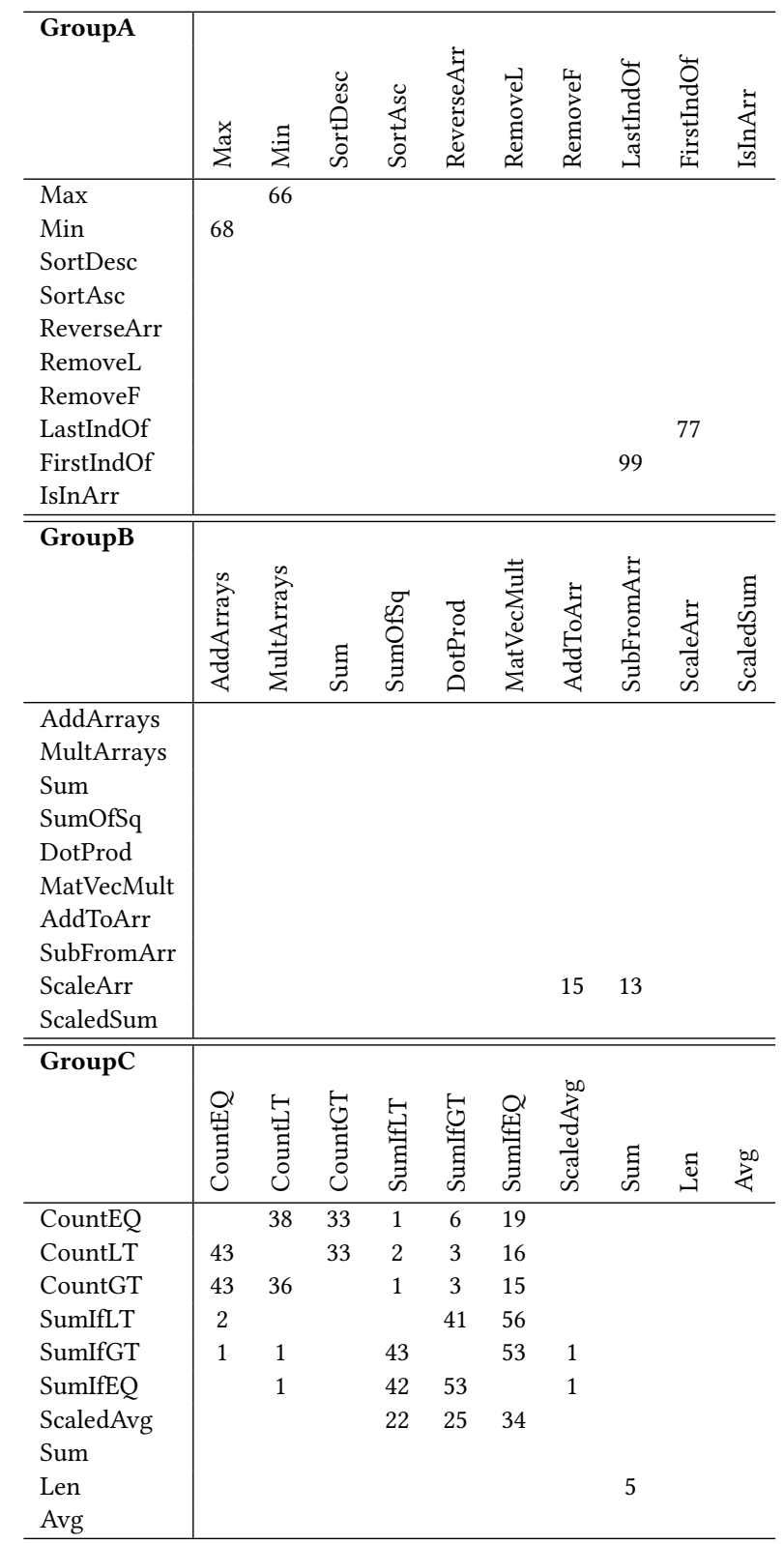

Table 3. Parent-Child relationships for GroupA, GroupB, and GroupC problems.

\section{Appendix D: Code Examples}

Code for each problem is given below. For brevity, only the main function is listed (i.e., the entire call tree is not listed for each solution). It should be noted that there are often many other solutions found in addition to the one shown.

\section{GroupA Problems}

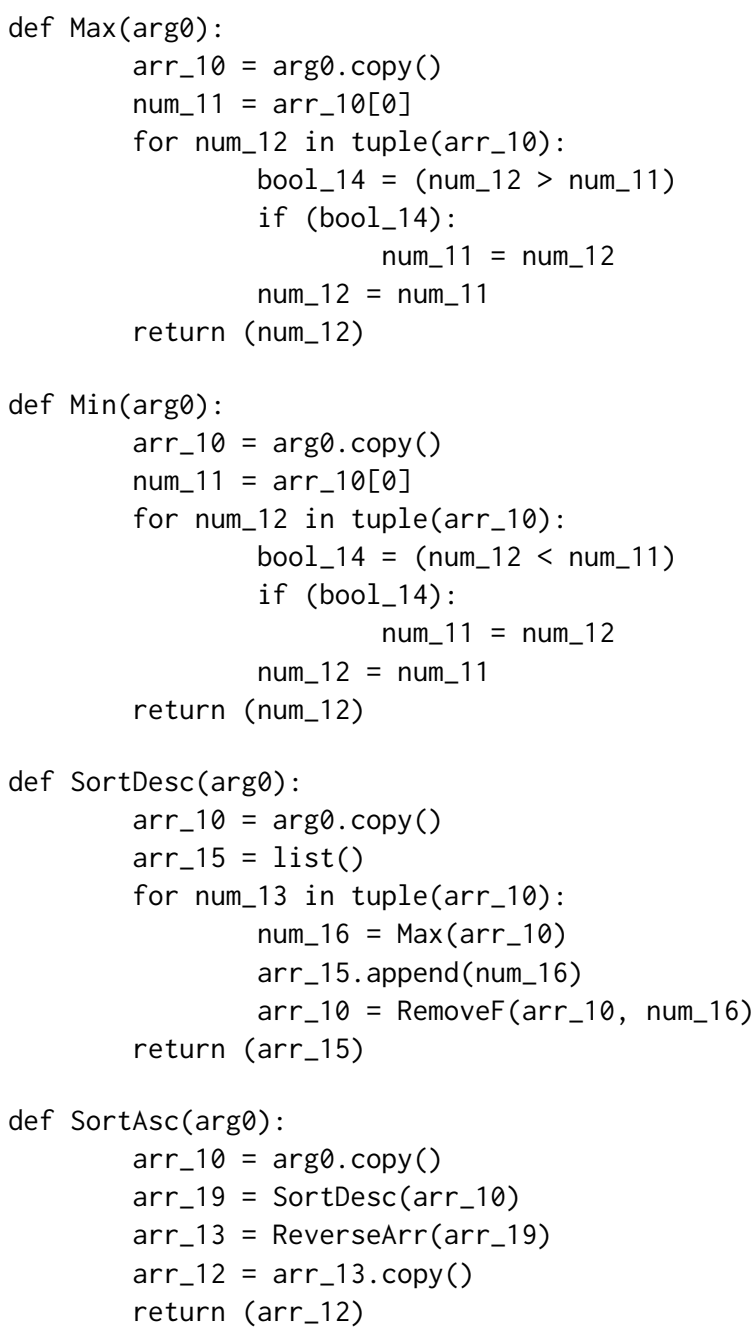


def RemoveF(arg0, arg1):

arr_10 $=\arg 0 . \operatorname{copy}()$

num_11 = arg1

num_17 = FirstInd0f (arr_10, num_11)

num_16 = arr_10.pop(num_17)

bool_12 = (num_17 $<$ num_16)

return (arr_10)

def LastInd0f(arg0, arg1):

arr_10 $=\arg 0 . \operatorname{copy}()$

num_11 = arg1

for num_14, num_15 in enumerate(tuple (arr_10)): bool_17 = (num_15 == num_11)

if (bool_17):

arr_10.append (num_14)

num_12 = arr_10.pop( $)$

return (num_12)

def Firstind0f (arg0, $\arg 1)$ :

arr_10 = arg0. $\operatorname{copy}()$

num_11 = arg1

arr_17 = list ()

for num_13, num_14 in enumerate(tuple $\left.\left(\operatorname{arr} \_10\right)\right)$ : bool_16 = (num_14 == num_11)

if (bool_16):

arr_17.append(num_13)

num_12 = arr_17[0]

return (num_12)

def IsInArr (arg0, arg1):

arr_10 $=\arg 0 . \operatorname{copy}()$

num_11 = arg1

for num_12 in tuple (arr_10):

bool_14 = (num_12 == num_11)

if (bool_14):

num_11 = arr_10[-1]

bool_13 = (num_12 == num_11)

return (bool_13)

\section{GroupB Problems}

def AddArrays (arg0, $\arg 1)$ :

arr_10 $=\arg 0 . \operatorname{copy}()$

arr_11 = arg1. $\operatorname{copy}()$

for num_13 in tuple(arr_10):

num_14 = arr_11.pop $(0)$

num_15 = num_13 + num_14

arr_11.append (num_15)

return (arr_11)

def Multarrays (arg0, $\arg 1)$ :

arr_10 $=\arg 0 \cdot \operatorname{copy}()$

arr_11 = arg1. copy ()

for num_13 in tuple(arr_10):

num_14 = arr_11.pop $(\theta)$

num_16 = arr_10.pop(0)

num_15 = num_16 $*$ num_14

arr_11.append (num_15) return (arr_11)

def $\operatorname{Sum}(\arg \theta)$ :

$\operatorname{arr} \_10=\arg 0 . \operatorname{copy}()$

num_14 = 0

for num_12 in tuple(arr_10):

num_14 = num_12 + num_14

return (num_14)

def SumOfSq(argo):

arr_10 = $\operatorname{arg0} . \operatorname{copy}()$

arr_14 = arr_10. copy ()

num_12 = DotProd(arr_14, arr_14)

return (num_12)

def DotProd(arg0, arg1):

arr_10 $=\arg 0 . \operatorname{copy}()$

arr_11 $=\arg 1 . \operatorname{copy}()$

arr_14 = MultArrays (arr_10, arr_11)

num_13 = Sum(arr_14)

return (num_13)

def MatVecMult (arg0, $\arg 1)$ :

arr_of_arr10 = arg0

arr_11 = arg1. $\operatorname{copy}()$

arr_16 = list ()

for arr_15 in tuple(arr_of_arr10): num_17 = DotProd(arr_11, arr_15) arr_16.append(num_17)

arr_14 = arr_16. $\operatorname{copy}()$

return (arr_14)

def AddToArr( $\arg 0, \arg 1)$ :

arr_10 $=\arg 0 . \operatorname{copy}()$

num_11 = arg1

for num_12 in tuple(arr_10):

num_15 = arr_10.pop $(0)$

num_14 = num_11 + num_15

arr_10. append(num_14)

return (arr_10)

def SubFromArr $(\arg 0, \arg 1)$ :

arr_10 $=\arg 0 . \operatorname{copy}()$

num_11 = arg1

for num_12 in tuple(arr_10):

num_13 = arr_10.pop(0)

num_14 = num_13 - num_11

arr_10.append (num_14)

return (arr_10)

def ScaleArr(arg0, $\arg 1)$ :

arr_10 $=\arg 0 . \operatorname{copy}()$

num_11 = arg1

arr_15 = list ()

for num_12 in tuple(arr_10):

num_14 = num_12 $*$ num_11

arr_15. append(num_14)

return (arr_15) 
def ScaledSum(arg0, arg1):

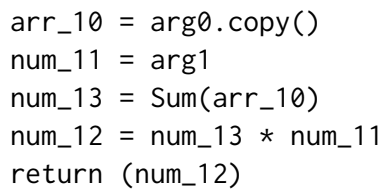

\section{GroupC Problems}

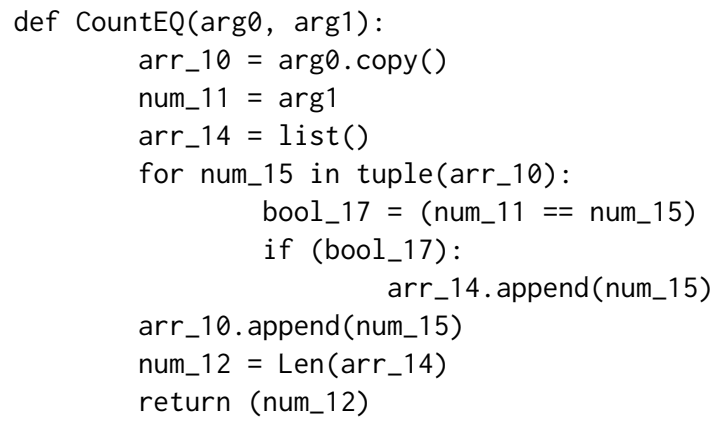

def SumIfGT( $\arg 0, \arg 1)$ :

arr_10 $=\arg 0 . \operatorname{copy}()$

num_11 = arg1

arr_14 = list ()

for num_13 in tuple(arr_10):

bool_15 = (num_11 >= num_13)

if (bool_15):

num_13 = 0

arr_14.append (num_13)

num_12 = Sum(arr_14)

return (num_12)

def SumIfEQ(arg0, $\arg 1)$ :

arr_10 $=\arg 0 . \operatorname{copy}()$

num_11 = arg1

arr_14 = list ()

for num_13 in tuple(arr_10): bool_15 = (num_11 != num_13) if (bool_15):

num_13 = 0

arr_14.append(num_13)

num_12 = Sum(arr_14)

return (num_12)

def ScaledAvg( $\arg 0, \arg 1)$ :

arr_10 $=\arg 0 . \operatorname{copy}()$

num_11 = arg1

for num_13 in tuple(arr_10): num_14 = arr_10.pop $(0)$

num_15 $=$ num_14 $*$ num_11

arr_10.append (num_15)

num_12 = Avg (arr_10)

return (num_12)

def Sum(argo):

arr_10 $=\arg 0 . \operatorname{copy}()$

num_17 $=0$

for num_12 in tuple (arr_10): num_17 = num_12 + num_17

return (num_17)

def Len( $\arg \theta)$ :

arr_10 $=\arg 0 . \operatorname{copy}()$

for num_14, num_15 in enumerate(tuple (arr_10)): pass

num_13 = 1

num_12 = num_13 + num_14

return (num_12)

def $\operatorname{Avg}(\arg \theta)$ :

arr_10 $=\arg 0 . \operatorname{copy}()$

arr_20 $=$ arr_10. copy ()

num_18 = Sum (arr_20)

num_17 = Len (arr_10)

num_13 = num_18 // num_17

num_12 = num_13

return (num_12) 


\section{Appendix E: Source Code \& Result Files}

\section{Source Code}

Please contact authors for source code (main.py). It can be run with Python 3.6 or later using the following command:

python main.py groupID

where, groupID is 1,2 , or 3, for GroupA, GroupB, and GroupC, respectively. The run prints progress on stdout and generates a report at the end (containing least-complex results, callees, parents, a stat record for each solution, etc.) in the current directory, a checkpoint in the ./chkpts directory, and a detailed log file for each rank in the ./log. nodename directory. On machines with fewer cores (than 112 we used), number of epochs must be increased proportionately for all solutions to be found.

After one or more such runs, one or more checkpoints can be read and least-complex solutions can be constructed for reporting purposes by running

python main.py groupID 1

Notice that least complex code produced in this step is composed from the least complex result found for each problem and the composed code is not currently tested using a Checker. This composed code is for reporting purposes only and must be inspected by the user.

\section{Result Files}

Please contact authors for result files. 